\title{
A NUMERICAL ESTIMATE OF THE REGULARITY OF A FAMILY OF STRANGE NON-CHAOTIC ATTRACTORS
}

\author{
LLUÍS ALSEDÀ I SOLER \\ JOSEP MARIA MONDELO GONZÁLEZ \\ AND \\ DAVID ROMERO I SÀNCHEZ
}

\begin{abstract}
We estimate numerically the regularities of a family of Strange Non-Chaotic Attractors related with one of the models studied in [GOPY84] (see also [Kel96]). To estimate these regularities we use wavelet analysis in the spirit of [dlLP02] together with some ad-hoc techniques that we develop to overcome the theoretical difficulties that arise in the application of the method to the particular family that we consider. These difficulties are mainly due to the facts that we do not have an explicit formula for the attractor and it is discontinuous almost everywhere for some values of the parameters. Concretely we propose an algorithm based on the Fast Wavelet Transform. Also a quality check of the wavelet coefficients and regularity estimates is done.
\end{abstract}

\section{INTRODUCTION}

The aim of this paper is to develop techniques and algorithms to compute approximations of (geometrically) extremely complicated dynamical invariant objects by means of wavelet expansions. Moreover, from the wavelet coefficients we want to derive an estimate of the regularity of these invariant objects. In the case when the theoretical regularity is known, the comparison between both values gives a natural and good quality test of the algorithms and approximations.

In this paper the invariant objects that we study and consider when developing our algorithms are Strange Non-chaotic Attractors. They appear in a natural way in families of quasiperiodically forced skew products on the cylinder of the form

$$
\begin{aligned}
& \mathfrak{F}_{\sigma, \varepsilon}: \mathbb{S}^{1} \times \mathbb{R} \longrightarrow \mathbb{S}^{1} \times \mathbb{R} \\
& (\theta, x) \quad \longmapsto \quad\left(R_{\omega}(\theta), F_{\sigma, \varepsilon}(\theta, x)\right),
\end{aligned}
$$

where $F_{\sigma, \varepsilon}: \mathbb{S}^{1} \times \mathbb{R} \longrightarrow \mathbb{R}$ is continuous and $\mathcal{C}^{1}$ with respect to the second variable, $R_{\omega}(\theta)=\theta+\omega(\bmod 1)$ with $\omega \in \mathbb{R} \backslash \mathbb{Q}, \mathbb{S}^{1}=\mathbb{R} / \mathbb{Z}=[0,1)$ denotes the circle and $\varepsilon, \sigma \in \mathbb{R}^{+}$. These systems have the important property that any fibre, $\{\theta\} \times \mathbb{R}$, is mapped into another fibre, $\left\{R_{\omega}(\theta)\right\} \times \mathbb{R}$.

Our main goal will be to derive approximations in terms of wavelets of the invariant maps $\varphi: \mathbb{S}^{1} \longrightarrow \mathbb{R}: \varphi\left(R_{\omega}(\theta)\right)=F_{\sigma, \varepsilon}(\theta, \varphi(\theta))$. Under certain conditions the graphs of these invariant maps have very complicated geometry where roughly speaking, the word complicated means non-piecewise continuous. In such case, we will say that the graph of $\varphi$ is a Strange Non-chaotic Attractor (SNA). A usual particular case of SNA is when the invariant function is positive in a set of full Lebesgue measure and vanishes on a residual set.

Date: November 20, 2016.

2010 Mathematics Subject Classification. Primary: 37M99, 37C55, 37C70, 42C40, 26A16, $30 \mathrm{H} 25$.

Key words and phrases. Wavelets, regularity, quasiperiodically forced system.

The authors have been partially supported by MINECO grant numbers MTM2008-01486, MTM2011-26995-C02-01 and MTM2014-52209-C2-1-P. 
A standard approach is to use Fourier expansions (rather than wavelet ones) when approximating dynamical invariant objects. In the SNA's framework this approach has a serious drawback: an accurate approximation of $\varphi$ demands a high number of Fourier modes due the appearance of strong oscillations (see e.g. [Jor01]). One natural way to overcome this problem is by using other orthonormal basis such as wavelets and the multi-scale methods (see e.g. [Coh03, Mal98]). One of the advantages of this approach is that wavelets also define certain regularity spaces $\mathscr{B}_{\infty, \infty}^{s}$ (see e.g [HW96, Coh03, Mey01, Tri06]) that provide a natural framework for the approximations that one gets.

Precisely, the regularity can be considered as a trait of how $\varphi$ becomes strange in terms of functional spaces. For example, in [dlLP02], the authors make numerical implementations of wavelet analysis to estimate the "positive" regularity of invariant objects which are graphs of functions in appropriate spaces. However, due to the complexity of the SNAs described above we need to consider the possibility that these objects have zero or even negative regularity (see [Coh03]). Hence, the techniques of [dlLP02] need to be extended to this case. To this end, we develop ad-hoc techniques to overcome the theoretical difficulties of the objects we study in performing a wavelet analysis, in the same spirit of [dlLP02], to estimate the regularity of such $\varphi$. Our wavelet analysis will be based on the Fast Wavelet Transform (see e.g [Mal98]).

The computation of the regularity (depending on parameters) can give some insight in the study of the fractalization or other routes of creation of SNA and help in detecting this bifurcation.

We apply the above program to a slight modification of the system considered in [GOPY84]. Indeed, the attractor obtained in [GOPY84] (as shown by Keller in $[$ Kel96]), is the graph of an upper semi-continuous function from the circle to $\mathbb{R}$ in the pinched case (that is, when there exists a fibre whose image is degenerate to a point), whereas in the non pinched one the attractor is the graph of a map with the same regularity as the skew product (see also [Sta97] and [Sta99]). As we will see, the wavelet coefficients together with the computed regularity detect well the functional space jump associated to the creation of the SNA.

This paper is organized in two parts. The first one is devoted to make a survey on wavelets and regularity. Whereas the second one deals with the application of these techniques to the SNA case. More concretely, in Section 2 we recall some topics about the theory of wavelet bases. In Section 3 we will review the notion of regularity through Besov functional spaces and discuss it by means of simple examples. In Besov spaces the regularity can be any real number (in contrast to Hölder regularity defined only for positive regularities). In Section 4 we survey the relation between the regularity and the wavelet coefficients of a function. Section 4.1 is devoted to present and test a methodology to numerically estimate regularities based on the previous sections.

Finally, in the second part, in Section 5 we present the family of Strange NonChaotic Attractors that we will study. In particular, we state Keller's Theorem and we emphasize some ideas on the proof. These ideas will be used in devising the algorithm that we propose. In Section 6 , we present the techniques to overcome the theoretical difficulties arising from the SNA. In 6.3, we perform the algorithm to compute the regularity of the attractors and in Section 7, the results of this computation, for a particular instance of SNA's, are presented and discussed.

Acknowledgements. We thank the two anonymous referees of the paper for his eager reading of the two versions of the paper and for pointing out some flaws and possible improvements of the paper that greatly helped us in clarifying some delicate arguments and improved the writing of the paper. 


\section{A SURVEY ON WAVELETS}

We aim at approximating by means of wavelets a certain class of functions from the circle $\mathbb{R} / \mathbb{Z}$ to an interval of the real line. Recall that a standard approach used in the literature to compute and work with invariant objects of systems exhibiting periodic or quasi-periodic behaviour is to use finite Fourier approximations (trigonometric polynomials), namely functions of the form

$$
\varphi(\theta)=a_{0}+\sum_{n=1}^{N}\left(a_{n} \cos (n \theta)+b_{n} \sin (n \theta)\right) .
$$

In this paper instead we aim at using finite wavelet expansions of the form:

$$
\varphi(\theta)=a_{0}+\sum_{j=0}^{J} \sum_{n=0}^{N_{j}} d_{j, n} \psi_{j, n}(\theta)
$$

where $\psi_{j, n}(\theta)$ is obtained by translation and dilation of a mother wavelet $\psi(x)$. To be explicit, let us start by introducing the orthonormal wavelet basis of $\mathscr{L}^{2}(\mathbb{R})$. A natural way to do it is via the notion of Multiresolution Analysis. We refer the reader to [Mal98, HW96] for more detailed and comprehensive expositions.

Definition 2.1. A sequence of closed subspaces $\left\{\mathcal{V}_{j}\right\}_{j \in \mathbb{Z}}$ of $\mathscr{L}^{2}(\mathbb{R})$ is a Multiresolution Analysis (or simply a $M R A$ ) if it satisfies the following six properties:

(a) $\{0\} \subset \cdots \subset \mathcal{V}_{1} \subset \mathcal{V}_{0} \subset \mathcal{V}_{-1} \subset \cdots \subset \mathscr{L}^{2}(\mathbb{R})$.

(b) $\{0\}=\bigcap_{j \in \mathbb{Z}} \mathcal{V}_{j}$.

(c) $\operatorname{clos}\left(\bigcup_{j \in \mathbb{Z}} \mathcal{V}_{j}\right)=\mathscr{L}^{2}(\mathbb{R})$.

(d) There exists a function $\phi$ whose integer translates, $\phi(x-n)$, form an orthonormal bases of $\mathcal{V}_{0}$. Such function is called the scaling function.

(e) For each $j \in \mathbb{Z}$ it follows that $f(x) \in \mathcal{V}_{j}$ if and only if $f\left(x-2^{j} n\right) \in \mathcal{V}_{j}$ for each $n \in \mathbb{Z}$.

(f) For each $j \in \mathbb{Z}$ it follows that $f(x) \in \mathcal{V}_{j}$ if and only if $f(x / 2) \in \mathcal{V}_{j+1}$.

Before continuing the explanation, let us recall that for $f \in \mathscr{L}^{2}(\mathbb{R})$,

$$
\widehat{f}(\xi)=\int_{\mathbb{R}} f(x) e^{-i \xi x} d x, \xi \in \mathbb{R},
$$

denotes the Fourier transform of $f$ and $f^{\vee}(x)$

$$
f^{\vee}(x)=\frac{1}{2 \pi} \int_{\mathbb{R}} f(\xi) e^{i \xi x} d \xi, x \in \mathbb{R}
$$

stands for the inverse Fourier transform. If we fix an MRA, it follows that $\mathcal{V}_{j}$ has an orthonormal basis $\left\{\phi_{j, n}\right\}_{n \in \mathbb{Z}}$, for every $j$, where

$$
\phi_{j, n}(x)=2^{-j / 2} \phi\left(\frac{x-2^{j} n}{2^{j}}\right) .
$$

Now, define the subspace $\mathcal{W}_{j}$ as the orthogonal complement of $\mathcal{V}_{j}$ on $\mathcal{V}_{j-1}$, that is,

$$
\mathcal{V}_{j-1}=\mathcal{W}_{j} \oplus \mathcal{V}_{j}
$$

Therefore, by the inclusion of the spaces $\mathcal{V}_{j}$ we have

$$
\mathscr{L}^{2}(\mathbb{R})=\operatorname{clos}\left(\bigoplus_{j \in \mathbb{Z}} \mathcal{W}_{j}\right)=\operatorname{clos}\left(\mathcal{V}_{0} \oplus \bigoplus_{j=-\infty}^{0} \mathcal{W}_{j}\right)
$$


The mother wavelet $\psi \in \mathcal{W}_{0}$ is defined to be the function whose Fourier transform is

$$
\widehat{\psi}(\xi)=\frac{1}{\sqrt{2}} e^{-i \xi} \widehat{h}^{*}(\xi+\pi) \widehat{\phi}(\xi)
$$

where $\widehat{h}^{*}(\xi)$ is the complex conjugate of

$$
\widehat{h}(\xi)=\sum_{n \in \mathbb{Z}} h[n] e^{-i n \xi},
$$

with $\widehat{h}(0)=\sqrt{2}$ and $h[n]=\left\langle\frac{1}{\sqrt{2}} \phi\left(\frac{x}{2}\right), \phi(x-n)\right\rangle$ for $n \in \mathbb{Z}$. The sequence $h[n]$ is called the scaling filter (or the low pass filter) of the Multiresolution Analysis. We define the support of $h[n]$, denoted by $\operatorname{supp}(h)$, as the minimum subset $\mathfrak{I}$ of $\mathbb{Z}$ such that $\mathfrak{I}=\left\{\ell, \ell+1, \ldots, \ell^{\prime}\right\}$ is a set of consecutive integers and

$$
h[n]=0 \text { for every } n \in \mathbb{Z} \backslash \mathfrak{I} .
$$

The following result (see [Mal98, Theorem 7.3]) allows to obtain the wavelet basis from the scaling function:

Theorem 2.2 (Mallat, Meyer). The mother wavelet given by equation (4) verifies that, for each integer $j$, the family $\left\{\psi_{j, n}\right\}_{n \in \mathbb{Z}}$ is an orthonormal basis of $\mathcal{W}_{j}$, where:

$$
\psi_{j, n}(x)=2^{-j / 2} \psi\left(\frac{x-2^{j} n}{2^{j}}\right) .
$$

As a consequence, the family $\left\{\psi_{j, n}\right\}_{(j, n) \in \mathbb{Z} \times \mathbb{Z}}$ is an orthonormal basis of $\mathscr{L}^{2}(\mathbb{R})$.

We want to approximate maps $f \in \mathscr{L}^{2}(\mathbb{R})$ by linear combinations of wavelets. By (3) and the theorem above, the projection of $f$ to $\mathcal{V}_{-J} \subset \mathscr{L}^{2}(\mathbb{R})$ :

$$
\sum_{n \in \mathbb{Z}}\left\langle f, \phi_{-J, n}\right\rangle \phi_{-J, n}
$$

is a good approximation of $f$ provided that $J>0$ is large enough. We want to rewrite such an approximation as linear combination of wavelets of the form

$$
f \sim \sum_{n \in \mathbb{Z}}\left\langle f, \phi_{0, n}\right\rangle \phi_{0, n}+\sum_{j=0}^{J-1} \sum_{n \in \mathbb{Z}}\left\langle f, \psi_{-j, n}\right\rangle \psi_{-j, n} \in \mathcal{V}_{0} \oplus \bigoplus_{j=0}^{J-1} \mathcal{W}_{j}
$$

To do it, as usual, we define the coefficients

$$
a_{j}[n]:=\left\langle f, \phi_{j, n}\right\rangle \quad \text { and } \quad d_{j}[n]:=\left\langle f, \psi_{j, n}\right\rangle
$$

for $j, n \in \mathbb{Z}$. With this notation, the initial approximation of $f$ becomes

$$
\sum_{n \in \mathbb{Z}} a_{-J}[n] \phi_{-J, n}
$$

By (2) we have

$$
\sum_{n \in \mathbb{Z}} a_{-j}[n] \phi_{-j, n}=\sum_{n \in \mathbb{Z}} a_{-j+1}[n] \phi_{-j+1, n}+\sum_{n \in \mathbb{Z}} d_{-j+1}[n] \psi_{-j+1, n}
$$

for every $j \in \mathbb{Z}$.

To obtain the coefficients $a_{-j+1}[n]$ and $d_{-j+1}[n]$ from $a_{-j}[n]$, we use the Fast Wavelet Transform (FWT) given by (see [Mal98, Theorem 7.7]):

$$
\left\{\begin{array}{l}
a_{j+1}[p]:=\sum_{n \in \mathbb{Z}} h[n-2 p] a_{j}[n] \quad \text { and } \quad d_{j+1}[p]:=\sum_{n \in \mathbb{Z}} g[n-2 p] a_{j}[n] \\
\text { with } g[p]=(-1)^{1-p} h[1-p]
\end{array}\right.
$$


for every $j, p \in \mathbb{Z}$. Hence, from the iterative use of (6) and (7) starting with the approximation $\sum_{n \in \mathbb{Z}} a_{-J}[n] \phi_{-J, n}$ we obtain the approximation of $f$ that we are looking for:

$$
f \sim \sum_{n \in \mathbb{Z}} a_{0}[n] \phi_{0, n}+\sum_{j=0}^{J-1} \sum_{n \in \mathbb{Z}} d_{-j}[n] \psi_{-j, n} \in \mathcal{V}_{0} \oplus \bigoplus_{j=0}^{J-1} \mathcal{W}_{j}
$$

For (numerical) applications such infinite approximations are usually not available since we often work with finite information about our function. For this we need a similar theory for subspaces of $\mathcal{V}_{j}$ and $\mathcal{W}_{j}$ of finite dimension. For $j \geq 0$ we define

$$
\begin{aligned}
\mathcal{V}_{-j}^{*} & :=\left\langle\phi_{-j, 0}, \phi_{-j, 1}, \ldots, \phi_{-j, 2^{j}-1}\right\rangle \subset \mathcal{V}_{-j}, \text { and } \\
\mathcal{W}_{-j}^{*} & :=\left\langle\psi_{-j, 0}, \psi_{-j, 1}, \ldots, \psi_{-j, 2^{j}-1}\right\rangle \subset \mathcal{W}_{-j},
\end{aligned}
$$

where $\left\langle f_{1}, f_{2}, \ldots, f_{n}\right\rangle$ denotes the subspace of $\mathscr{L}^{2}(\mathbb{R})$ generated by the linear combinations of $f_{1}, f_{2}, \ldots, f_{n}$. From the comment at the end of Section 7.3.1 of [Mal98] (see also [Fra99, Lemma 3.26] for a more detailed account), it follows that

$$
\mathcal{V}_{-j+1}^{*}=\mathcal{W}_{-j}^{*} \oplus \mathcal{V}_{-j}^{*}
$$

for every $j>0$. Hence, given a function $f \in \mathscr{L}^{2}(\mathbb{R})$ we can take a good finite approximation of the map given by its projection to $\mathcal{V}_{-J}^{*}$ :

$$
f \sim \sum_{n=0}^{2^{J}-1} a_{-J}[n] \phi_{-J, n}
$$

provided that $J$ is large enough. Again, we are interested in writing such an approximation as linear combination of wavelets, but in this case this expansion must be finite:

$$
f \sim a_{0}[0] \phi_{0,0}+\sum_{j=0}^{J-1} \sum_{n=0}^{2^{j}-1} d_{-j}[n] \psi_{-j, n} \in \mathcal{V}_{0}^{*} \oplus \bigoplus_{j=0}^{J-1} \mathcal{W}_{-j}^{*} .
$$

To obtain this expression observe that (8) implies

$$
\sum_{n=0}^{2^{j}-1} a_{-j}[n] \phi_{-j, n}=\sum_{n=0}^{2^{j-1}-1} a_{-j+1}[n] \phi_{-j+1, n}+\sum_{n=0}^{2^{j-1}-1} d_{-j+1}[n] \psi_{-j+1, n}
$$

for $j>0$. Now, to obtain the coefficients $a_{-j+1}[n]$ and $d_{-j+1}[n]$ from $a_{-j}[n]$, instead of using formulae (7), we use the following circular convolution version of them (see [Mal98, Section 7.5.1] or the proof of [Fra99, Lemma 3.26]):

$$
\left\{\begin{array}{l}
a_{-j+1}[p]:=\sum_{n=0}^{2^{j}-1} h[n-2 p] a_{-j}[n] \quad \text { and } \quad d_{-j+1}[p]:=\sum_{n=0}^{2^{j}-1} g[n-2 p] a_{-j}[n] \\
\text { with } g[p]=(-1)^{1-p} h[1-p]
\end{array}\right.
$$

for every $j>0$ and $p \in\left\{0,1, \ldots, 2^{j-1}-1\right\}$. Hence, with the iterative use of (10) and (11) starting with the approximation (9) we obtain

$$
f \sim a_{0}+\sum_{j=0}^{J-1} \sum_{n=0}^{2^{j}-1} d_{-j}[n] \psi_{-j, n} \in \mathcal{V}_{0}^{*} \oplus \bigoplus_{j=0}^{J-1} \mathcal{W}_{-j}^{*}
$$

as we wanted.

To effectively compute an approximation of the type given in equation (12) one remaining problem is left: to find a good estimate of the initial coefficients $a_{-J}[n]=\left\langle f, \phi_{-J, n}\right\rangle$. In the literature there is a lot of discussion on how to compute 
these coefficients, but a simple customary approach is to use the following estimate (see, for instance, [Fra99, Lemma 5.54] and its proof):

Lemma 2.3. Assume that $f$ verifies $\left|\left\langle f, \phi_{j, n}\right\rangle\right|<\infty$ for every $j, n \in \mathbb{Z} \times \mathbb{Z}$ and

$$
|f(x)-f(y)| \leq C_{1}|x-y|^{\alpha} \text { with } \alpha \in(0,1]
$$

for all real numbers $x, y$ and a constant $C_{1}<\infty$. Suppose that the scaling function $\phi$ from an $M R A\left\{\mathcal{V}_{j}\right\}_{j \in \mathbb{Z}}$ is such that

$$
\phi \in \mathscr{L}^{1}(\mathbb{R}), \widehat{\phi}(0)=\int_{\mathbb{R}} \phi(x) d x=1 \text { and } \int_{\mathbb{R}}|x|^{\alpha} \phi(x) d x<C_{2} .
$$

Then, for every $j, n \in \mathbb{Z} \times \mathbb{Z}$,

$$
\left|\left\langle f, \phi_{j, n}\right\rangle-2^{j / 2} f\left(2^{j} n\right)\right|<C_{1} C_{2} 2^{j\left(\alpha+\frac{1}{2}\right)} .
$$

As a corollary of this lemma we see that if $f$ is Lipschitz, then

$$
a_{-J}[n] \approx 2^{-J / 2} f\left(2^{-J} n\right) .
$$

Summarizing, Lemma 2.3 gives us a method to initialize the FWT. This gives a complete algorithm to compute wavelet coefficients of a certain function $f \in \mathscr{L}^{2}(\mathbb{R})$.

\section{Defining Regularity through Besov spaces}

In this section we will make precise the notion of regularity that we will use. To do so, we will describe, in two steps, the functional spaces that define the notion of regularity. Roughly speaking these spaces collect the functions that verify an $\alpha$-Hölder condition. However, as we will see, we will have to deal with functions with regularity zero (that do not verify any Hölder condition). The notion of nonpositive regularity is formalized trough Besov spaces (see [Tri83, BL76]). In what follows, we will recall the definition of the Besov spaces on the real line [Tri83, Section 2.3] and the extension of such definition to $\mathbb{S}^{1}$.

3.1. The spaces $\mathscr{B}_{\infty, \infty}^{s}(\mathbb{R})$. The space of all real valued rapidly decreasing infinitely differentiable functions is called the (real) Schwartz space and it is denoted by $\mathcal{S}(\mathbb{R})$. The topological dual of $\mathcal{S}(\mathbb{R})$ is the space of tempered distributions which is denoted by $\mathcal{S}^{\prime}(\mathbb{R})$. For $f \in \mathcal{S}^{\prime}(\mathbb{R}), \widehat{f}(\xi)$ denotes the Fourier transform of $f$ and $f^{\vee}(x)$ stands for the inverse Fourier transform in the sense of distributions (see e.g [Tri83]). Recall, also, that the essential supremum is defined as

$$
\underset{x \in \mathbb{R}}{\operatorname{ess} \sup } f(x)=\inf \{a \in \mathbb{R}: \mu(\{x \in \mathbb{R}: f(x)>a\})=0\},
$$

where $\mu$ is a measure (in our case the usual Lebesgue measure).

Let $\varphi_{0} \in \mathcal{S}(\mathbb{R})$ be such that

$$
\varphi_{0}(x):= \begin{cases}1 & \text { if }|x| \leq 1 \\ 0 & \text { if }|x| \geq 3 / 2\end{cases}
$$

and set

$$
\varphi_{j}(x):=\varphi_{0}\left(2^{-j} x\right)-\varphi_{0}\left(2^{-j+1} x\right)
$$

for $j \in \mathbb{N}$. It is not difficult to show that, independently of the choice of $\varphi_{0}$, $\sum_{j=0}^{\infty} \varphi_{j}(x)=1$ for all $x \in \mathbb{R}$. Each of the families $\left\{\varphi_{j}\right\}_{j=0}^{\infty}$ is called a Dyadic Resolution of Unity in $\mathbb{R}$.

Definition 3.1. Let $\varphi=\left\{\varphi_{j}\right\}_{j=0}^{\infty}$ be a Dyadic Resolution of Unity and $s \in \mathbb{R}$. For $f \in \mathcal{S}^{\prime}(\mathbb{R})$ we define the quasi-norm

$$
\|f\|_{\infty, \infty, \varphi, s}=\sup _{j \geq 0} 2^{j s}\left(\underset{x \in \mathbb{R}}{\operatorname{ess} \sup _{0}}\left|\left(\varphi_{j} \widehat{f}\right)^{\vee}(x)\right|\right) .
$$


Then, we define the Besov Spaces by

$$
\mathscr{B}_{\infty, \infty}^{s}(\mathbb{R}):=\left\{f \in \mathcal{S}^{\prime}(\mathbb{R}):\|f\|_{\infty, \infty, \varphi, s}<\infty\right\}
$$

As it can be seen in [Tri83, Remark 2 of Section 2.3], the spaces $\mathscr{B}_{\infty, \infty}^{s}(\mathbb{R})$ are, in fact, independent of the chosen dyadic resolution of unity $\varphi$. Therefore, we can remove the subscript $\varphi$ from $\|f\|_{\infty, \infty, \varphi, s}$. So, in what follows we will write $\|f\|_{\infty, \infty, s}$ instead of $\|f\|_{\infty, \infty, \varphi, s}$. The spaces $\mathscr{B}_{\infty, \infty}^{s}(\mathbb{R})$ are a particular case of the Generalized Besov Spaces $\mathscr{B}_{p, q}^{s}(\mathbb{R})$ defined also, for example, in [Tri83] and one has the inclusion property $\mathscr{B}_{p, q}^{s^{\prime}}(\mathbb{R}) \subset \mathscr{B}_{p, q}^{s}(\mathbb{R})$ when $s<s^{\prime}$.

Remark 3.2. From [Tri83] it follows that the spaces $\mathscr{B}_{\infty, \infty}^{s}(\mathbb{R})$ with $s>0$ coincide with the corresponding Hölder-Zygmund spaces $\mathcal{C}^{s}(\mathbb{R})$.

From the above remark it is natural to extend the notion of regularity to $s \leq$ 0 through $\mathscr{B}_{\infty, \infty}^{s}(\mathbb{R})$ in the following way (we refer to [Tri83, Ste70] for a more complete explanation).

Definition 3.3. We say that a map $f$ has regularity $s \in \mathbb{R}$ if $f \in \mathscr{B}_{\infty, \infty}^{s}(\mathbb{R})$.

Example 3.4. The following examples help to clarify this regularity notion.

(i) Consider the Weierstraß function defined by

$$
\mathfrak{W}_{A, B}(x):=\sum_{n=1}^{\infty} A^{n} \sin \left(B^{n} x\right),
$$

where $A, B \in \mathbb{R}$ are such that $B^{-1}<A<1$ is Hölder continuous and nowhere differentiable (but it has a distributional derivative, as does every locally integrable function). Moreover, it has regularity $-\log _{B}(A)$; that is $\mathfrak{W}_{A, B} \in \mathscr{B}_{\infty, \infty}^{-\log _{B}(A)}(\mathbb{R})$.

(ii) The function $f(x)=\frac{-1}{\log (|x|)}$ (with $f(0)=0$ ) belongs to $\mathscr{B}_{\infty, \infty}^{0}$ in a neighbourhood of $x=0$ since $F(x)$, where $F^{\prime}(x)=f(x)$, is Lipschitz (because it is an anti-derivative of a bounded function) and the derivative operator reduces $s$ by 1 (leaving $p=q=\infty$ unchanged). Examples of functions belonging to different functional spaces are given in [RS96, Section 2.3, Example 1]. The prototypical example is to consider $\alpha^{2}+\beta^{2}>0$, with $\beta>0$, and defining

$$
f_{\alpha, \beta}(x)=v(x)|x|^{\alpha}(-\log |x|)^{-\beta}
$$

where $v(x)$ is a smooth cut-off function with $\operatorname{supp} v \subset\{x \in \mathbb{R}:|x| \leq \delta\}$ and $\delta>0$.

(iii) In [Coh03, Section 3.8] it is shown that, for $s>0, \mathscr{B}_{\infty, \infty}^{-s}$ is the dual of $\mathscr{B}_{\infty, \infty}^{s}$. That is, the spaces $\mathscr{B}_{\infty, \infty}^{-s}$ are "purely" distributional spaces. For example, it is known that $\delta(x) \in \mathscr{B}_{\infty, \infty}^{-1}(\mathbb{R})$ where $\delta(x)$ stands for Dirac's delta. In view of that, $\delta(x)$ can be considered as the second (distributional) derivative of the continuous function

$$
f(x)= \begin{cases}0 & \text { if } x<0 \\ x & \text { if } x \geq 0\end{cases}
$$

We conclude this subsection with some remarks on the spaces $\mathscr{B}_{\infty, \infty}^{s}$. The fact that $f \in \mathscr{B}_{\infty, \infty}^{s}$ with $s>0$ is equivalent to specify its differentiability degree and how "wild" is the last derivative. Indeed, if $s \in(0,1)$ then, $|f(x+h)-f(x)|<$ $C|h|^{s}$ for all $x \in \mathbb{R}$ and $h$ in an open neighbourhood of 0 . On the other hand, 
if $s=n+\alpha$, where $n \in \mathbb{N}$ and $\alpha \in(0,1)^{1}$, then $f$ is $\mathcal{C}^{n}$ and $f^{n)} \in \mathscr{B}_{\infty, \infty}^{\alpha}$. Consequently, when $n \geq 1, f$ is continuously differentiable whereas if $n=0$ then we have

Proposition 3.5 (Proposition V.4.6 [Ste70]). Every $f \in \mathscr{B}_{\infty, \infty}^{s}(\mathbb{R})$, with $s \in(0,1)$ may be modified on a set of measure zero so that it becomes continuous.

Finally, we want to analytically compute the regularity of the upper semi-continuous functions. This will be useful when developing the quality control of our algorithm.

Lemma 3.6. Let $f \in \mathscr{L}^{2}(\mathbb{R})$ be an upper semi-continuous function which is not continuous. Then $f \in \mathscr{B}_{\infty, \infty}^{0}(\mathbb{R})$ (that is, $f$ has zero regularity).

Proof. If $f$ is an upper semi-continuous function which is not continuous then, it cannot belong to $\mathscr{B}_{\infty, \infty}^{s}$ with $s>0$. On the other side, since $f$ is not a distribution, by [Coh03, Section 3.8], the parameter $s$ cannot be negative. Thus, $f \in \mathscr{B}_{\infty, \infty}^{0}(\mathbb{R})$.

3.2. The Besov spaces on $\mathbb{S}^{1}$. In this section we will extend the Besov spaces to $\mathbb{S}^{1}$. Recall that we consider $\mathbb{S}^{1}=\mathbb{R} / \mathbb{Z}$ and, hence, as the interval $[0,1)$. To do it we follow [BL76, Tri92]. Indeed, given $f \in \mathcal{S}^{\prime}\left(\mathbb{S}^{1}\right)$ (the space of tempered distributions on $\left.\mathbb{S}^{1}\right)$ it is known that

$$
f=\sum_{n \in \mathbb{Z}} \widehat{f}(n) e^{i n x}
$$

Definition 3.7. Let $\varphi=\left\{\varphi_{j}\right\}_{j=0}^{\infty}$ be a dyadic resolution of unity (on $\mathbb{R}$ ). We define the Besov Spaces on $\mathbb{S}^{1}$ by

$$
\mathscr{B}_{\infty, \infty}^{s}\left(\mathbb{S}^{1}\right):=\left\{f \in \mathcal{S}^{\prime}\left(\mathbb{S}^{1}\right):\|f\|_{\infty, \infty, s}<\infty\right\}
$$

where

$$
\|f\|_{\infty, \infty, s}=\sup _{j \geq 0} 2^{j s}\left(\underset{x \in \mathbb{R}}{\operatorname{ess} \sup }\left|\sum_{n \in \mathbb{Z}} \varphi_{j}(n) \widehat{f}(n) e^{i n x}\right|\right)
$$

is a quasi-norm for the quasi-Banach space $\mathscr{B}_{\infty, \infty}^{s}\left(\mathbb{S}^{1}\right)$.

As in Definition 3.3 we say that a circle map $f$ has regularity $s \in \mathbb{R}$ if the map $f$ belongs to $\mathscr{B}_{\infty, \infty}^{s}\left(\mathbb{S}^{1}\right)$.

The following lemma shows that the regularity of a circle map coincides with the regularity of its real extension which we define as follows. Given $f \in \mathcal{S}^{\prime}\left(\mathbb{S}^{1}\right)$ there exists a unique $f^{\text {PER }} \in \mathcal{S}^{\prime}(\mathbb{R})$ such that $f^{\text {PER }}$ is 1 -periodic and the restriction of $f^{\text {PER }}$ over $[0,1)$ coincides with $f$ (such an $f^{\text {PER }}$ can be defined as $f(\{\cdot\})$, where $\{\cdot\}$ denotes the fractional part function). This lemma is usually omitted and used implicitly but we include here for completeness.

Lemma 3.8. For every $f \in \mathcal{S}^{\prime}\left(\mathbb{S}^{1}\right)$ it follows that $f^{\mathrm{PER}} \in \mathscr{B}_{\infty, \infty}^{s}(\mathbb{R})$ if and only if $f \in \mathscr{B}_{\infty, \infty}^{s}\left(\mathbb{S}^{1}\right)$.

Proof. Since $f^{\mathrm{PER}}$ is 1-periodic and $\left.f^{\mathrm{PER}}\right|_{[0,1]}=f$,

$$
\widehat{f^{\mathrm{PER}}}(n)=\int_{0}^{1} f^{\mathrm{PER}}(x) e^{-2 \pi i n x} d x=\int_{0}^{1} f(x) e^{-2 \pi i n x} d x=\widehat{f}(n) .
$$

\footnotetext{
${ }^{1}$ We are not interested in the case $f \in \mathscr{B}_{\infty, \infty}^{n}$ with $n \in \mathbb{N}$. However we should say that it is known that there are Lipschitz functions that are not Zygmund functions. Therefore, the space $\mathscr{B}_{\infty, \infty}^{n}$ can be understood as a space that includes the $n$-Hölder and the $n$-Zygmund spaces (see [Tri83]).
} 
Hence,

$$
\begin{aligned}
& \operatorname{ess}_{x \in \mathbb{R}}\left|\sum_{n \in \mathbb{Z}} \varphi_{j}(n) \widehat{f}(n) e^{i n x}\right|=\operatorname{ess}_{x \in \mathbb{R}}\left|\sum_{n \in \mathbb{Z}} \varphi_{j}(n) \widehat{f^{\mathrm{PER}}}(n) e^{i n x}\right| \\
& =\operatorname{esssup}_{x \in \mathbb{R}}\left|\sum_{n \in \mathbb{Z}}\left(\varphi_{j} \widehat{f^{\mathrm{PER}}}\right)(n) e^{i n x}\right| \\
& =\underset{x \in \mathbb{R}}{\operatorname{ess} \sup _{0}}\left|\left(\varphi_{j} \widehat{f^{\mathrm{PER}}}\right)^{\vee}(x)\right| .
\end{aligned}
$$

That is, $\left\|f^{\text {PER }}\right\|_{\infty, \infty, s}=\|f\|_{\infty, \infty, s}$ and, hence, $f^{\text {PER }} \in \mathscr{B}_{\infty, \infty}^{s}(\mathbb{R})$ if and only if $f \in \mathscr{B}_{\infty, \infty}^{s}\left(\mathbb{S}^{1}\right)$.

\section{WAVELETS AND REGULARITY}

In Section 3 we have recalled the notion of the regularity of a function through the spaces $\mathscr{B}_{\infty, \infty}^{s}(\mathbb{R})$ and $\mathscr{B}_{\infty, \infty}^{s}\left(\mathbb{S}^{1}\right)$. Also, we have introduced the wavelet expansions of a given function in $\mathscr{L}^{2}(\mathbb{R})$. Next, we want to show the relationship between this notion of regularity and the wavelet coefficients. Such relationship will be the main tool of the forthcoming Algorithm 6.6. The main tool for this will be the Daubechies wavelets, because they are orthonormal bases on $\mathscr{L}^{2}(\mathbb{R})$ (see [Mal98] for a definition and construction) and, depending on the number of vanishing moments, they are well adapted to the functional spaces $\mathscr{B}_{\infty, \infty}^{s}(\mathbb{R})$ (see [HW96, Tri06]).

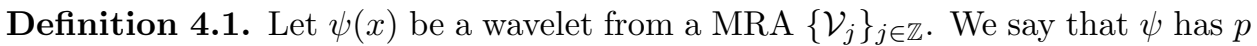
vanishing moments if the integer $p$ is the maximum non-negative integer such that

$$
\int_{\mathbb{R}} x^{k} \psi(x) d x=0 \text { for } 0 \leq k<p .
$$

Daubechies wavelets are a family of wavelets with compact support that have an element with $p$ vanishing moments for each $p \geq 1$. As it is customary we will denote them by $D 2 p$, where $p$ is the number of vanishing moments. From [HW96, Theorem 7.16], [Coh03, Theorem 3.8.1] and [Tri06, Theorem 1.64] we will state the following theorem, in the spirit of [dlLP02, Theorem 5.10] and [Mey01, Chapter 3], which will be useful for our purposes. To this end, for $t \in \mathbb{R}$ we set

$$
\mathrm{R}(t)= \begin{cases}t-\frac{1}{2} & \text { if } t>\frac{1}{2} \\ t+\frac{1}{2} & \text { if } t<-\frac{1}{2} \\ 0 & \text { if } t \in\left[-\frac{1}{2}, \frac{1}{2}\right] .\end{cases}
$$

Theorem 4.2. Let $f \in \mathscr{L}^{2}(\mathbb{R})$ and let $\psi$ be a mother Daubechies wavelet with more than $\max \left(\mathrm{R}(\tau), \frac{5}{2}-\mathrm{R}(\tau)\right)$ vanishing moments for some $\tau \in \mathbb{R} \backslash\left[-\frac{1}{2}, \frac{1}{2}\right]$. Then, $f \in \mathscr{B}_{\infty, \infty}^{\mathrm{R}(\tau)}(\mathbb{R})$ if and only if there exists $C>0$ such that

$$
\sup _{n \in \mathbb{Z}}\left|\left\langle f, \psi_{j, n}\right\rangle\right| \leq C 2^{\tau j}
$$

for all $j \leq 0$. Furthermore, if $\psi$ has more than 2 vanishing moments, then $f \in \mathscr{B}_{\infty, \infty}^{0}(\mathbb{R})$ if and only if either the sequence $\left\{2^{-\tau j} \sup _{n \in \mathbb{Z}}\left|\left\langle f, \psi_{j, n}\right\rangle\right|\right\}_{j=0}^{-\infty}$ is unbounded for every $\tau \in \mathbb{R}$ or there exist $C>0$ and $\tau \in\left[-\frac{1}{2}, \frac{1}{2}\right]$ such that

$$
\sup _{n \in \mathbb{Z}}\left|\left\langle f, \psi_{j, n}\right\rangle\right| \leq C 2^{\tau j}
$$

for $j \leq 0$ 
Remark 4.3. In view of Theorem 4.2, if the coefficients $\sup _{n \in \mathbb{Z}}\left|\left\langle f, \psi_{j, n}\right\rangle\right|$ decay approximately exponentially with respect to $j$, that is

$$
s_{j}:=\log _{2}\left(\sup _{n \in \mathbb{Z}}\left|\left\langle f, \psi_{j, n}\right\rangle\right|\right) \approx \tau j+\log _{2}(C),
$$

then $f \in \mathscr{B}_{\infty, \infty}^{\mathrm{R}(\tau)}(\mathbb{R})$. This tells us that, in this case, to compute the value of regularity $s$ we can make a linear regression to estimate the slope $\tau$ of the graph of the pairs $\left(j, s_{j}\right)$ and get the correct value of $s$ from this slope.

Moreover, in Theorem 4.2 the constant $C>0$ must be understood as the norm of $f$. Therefore, equation (13), can be used to see the transition between different Besov spaces. In other words, the passing from a concrete Besov space to another one can be recovered by the inspection of the values of $C$. This is because if $f \notin \mathscr{B}_{\infty, \infty}^{s}$ for some $s$ then the constant $C>0$ from equation (13) does not exist.

In view of the above Remark, we can perform a strategy to estimate the regularity of a function using the wavelet coefficients. This is the main topic of the following subsection.

4.1. A method to estimate regularities on $\mathscr{L}^{\infty}\left(\mathbb{S}^{1}\right)$. As we have said, we want to compute wavelet approximations of certain dynamical objects while controlling the precision of these approximations (i.e., the accuracy of the computed wavelet coefficients). This quality test will be done by comparing the theoretical regularity of such functions with the estimated one (given by Theorem 4.2 and Remark 4.3). More concretely, in [dlLP02], numerical implementations of wavelet analysis to estimate the positive regularity of conjugacies between critical circle maps are done. Due to Theorem 4.2, we can generalize such techniques to any value (positive or not) of the regularity measured in terms of the Besov Spaces $\mathscr{B}_{\infty, \infty}^{s}(\mathbb{R})$. The steps described below explain how to apply Theorem 4.2 in a general framework.

Among the many methods described in the literature to compute wavelet approximations, in this paper we will use (and test) the Fast Wavelet Transform. Alternatively, in a forthcoming paper we will explore the technique of solving numerically the Invariance Equation given in Remark 6.7 which can be more adapted to the dynamical complexity of the object.

Remark 4.4. In view of Lemma 3.8, to estimate the regularity of a map $f \in$ $\mathcal{S}^{\prime}\left(\mathbb{S}^{1}\right)$ it is enough to use Theorem 4.2 for $f^{\text {PER }}$. Moreover, if $f \in \mathscr{L}^{\infty}\left(\mathbb{S}^{1}\right)$, then $\left|\left\langle f^{\mathrm{PER}}, \psi_{j, n}\right\rangle\right|<\infty$ for all $j, n \in \mathbb{Z} \times \mathbb{Z}$.

In view of this remark, the verbatim application of Theorem 4.2 with a Daubechies wavelet with $k$ vanishing moments is the following:

Step 1. Fix a $J$ as large as possible and use Lemma 2.3 to compute $a_{-J}^{\mathrm{PER}}[n]:=$ $\left\langle f^{\mathrm{PER}}, \phi_{-J, n}\right\rangle$ for $0 \leq n \leq 2^{J}-1$.

Step 2. Use equation (7) to calculate the coefficients $d_{-j}^{\mathrm{PER}}[n]=\left\langle f^{\mathrm{PER}}, \psi_{-j, n}\right\rangle$ for $j=0, \ldots, J-1$ and $0 \leq n \leq 2^{j}-1$.

Step 3. By using the coefficients $d_{-j}^{\mathrm{PER}}[n]$ from Step 2, calculate, in view of Theorem 4.2 ,

for $j=0, \ldots, J-1$.

$$
s_{-j}=\log _{2}\left(\sup _{0 \leq n \leq 2^{j}-1}\left|d_{-j}^{\mathrm{PER}}[n]\right|\right)
$$

Step 4. In view of equation (13), make a linear regression to estimate the slope $\tau$ of the graph of the pairs $\left(-j, s_{-j}\right)$ with $j=0, \ldots, J-1$. Then, when there is evidence of linear correlation between the variables $-j$ and $s_{-j}$, we set $s=\mathrm{R}(\tau)$. 
Step 5. If $k>\max (s, 5 / 2-s)$ then, by Theorem $4.2, f^{\mathrm{PER}} \in \mathscr{B}_{\infty, \infty}^{s}(\mathbb{R})$ and, hence, $f$ has regularity $s$. Otherwise we need to repeat Step $1-4$ with a Daubechies wavelet having a larger value of $k$ until $k>\max (s, 5 / 2-s)$.

Remark 4.5 (On the quality of the linear model). The choice of $J$ has a direct influence on the estimated regularity which is derived from the slope of the straight line which better fits the linear model (Step 4). We have several comments on the influence of the choice of $J$ on the estimation of the regularity:

(i) Ideally one should find a $J>0$ large enough so that the projection of $f \in$ $\mathscr{L}^{2}\left(\mathbb{S}^{1}\right)$ into $\mathcal{V}_{-J}^{*}$ is good enough. More precisely, formula (3) with 0 replaced by $-J$, and Theorem 4.2 give the following formula for the truncation error from equation (9):

$$
\begin{aligned}
\left\|f^{\mathrm{PER}}-\sum_{n=0}^{2^{J}-1} a_{-J}^{\mathrm{PER}}[n] \phi_{-J, n}(x)\right\|_{2} & \leq\left\|\sum_{j=J}^{\infty} \sum_{n=0}^{2^{j}-1} d_{-j}^{\mathrm{PER}}[n] \psi_{-j, n}\right\|_{2} \\
& \leq C \sum_{j=J}^{\infty} 2^{-\tau j} \sum_{n=0}^{2^{j}-1}\left\|\psi_{-j, n}\right\|_{2}
\end{aligned}
$$

Then, $J>0$ should be large enough so that this truncation error is smaller than a given tolerance. However, notice that this estimate depends on the decay of the wavelet coefficients corresponding to the spaces $\mathcal{W}_{-j}$ with $j>J$ and, in particular, on the constant $C$ from Theorem 4.2. In other words, the truncation error from equation (9) depends on the regularity of the map $f$.

Moreover, by using Lemma 2.3 we can approximate the coefficients $a_{-J}^{\mathrm{PER}}[n]$ by $2^{-J / 2} f\left(2^{-J} n\right)$, with error

$$
\left|a_{-J}^{\mathrm{PER}}[n]-2^{-J / 2} f\left(2^{-J} n\right)\right|<C_{1} C_{2} 2^{-J\left(\alpha+\frac{1}{2}\right)} .
$$

Observe that, again, the constant $C_{1}$ depends on the regularity of $f$ (see Lemma 2.3 and the comments after Example 3.4).

Since it is not reasonable to assume that the regularity is known (specially when we are developing an algorithm to compute it) we are forced to heuristically fix the value of $J$ as the biggest that our computing resources and computation time can support.

(ii) Notice that Step 4 also has an influence on the choice of $J>0$. Indeed, from Theorem 4.2 we get $J$ samples to perform a linear regression with the pairs $\left(-j, s_{-j}\right)$ with $j=0, \ldots, J-1$. Therefore, one must take $J>0$ as big as possible to increase the reliability of the regressions on $J$ samples.

(iii) Moreover, among all samples $\left(-j, s_{-j}\right)$ with $j=0, \ldots, J-1$, one should only use the pairs $\left(-j, s_{-j}\right)$ where the linear dependence is clearly seen. Indeed, typically there is a region corresponding to small values of $|j|$ where certain properties of $f$ (e.g. self-similarity) are not displayed and, hence, we cannot expect linearity of the pairs $\left(-j, s_{-j}\right)$ (see Figure 2).

On the other hand, in the region corresponding to big values of $|j|$, the computed values of $s_{j}$ can become corrupt due to numerical errors. Moreover, since the wavelet basis elements at this scales have a very small support, every wavelet basis element "sees" very few samples of the $2^{J}$ initial samples of our function $f$. Hence, it is not reasonable to expect high precision at wavelet coefficients corresponding to small scales.

So, there is an intermediate zone where the linear dependence (compatible with the theory) it is observable. Thus, one should specify the (smaller) range of $j$ 's which is used in the computations. This still adds a new limitation to 
the number of samples available for the linear regressions which adds more importance to the need of taking $J>0$ as large as possible.

In view of the above remark, along our computations we will fix $J=30$. To test the precision of this implementation of Theorem 4.2 we will use the Weierstraß function. The reason for such experiment is twofold. From one side there exists an analytic formula for the regularity of the Weierstraß function in terms of its parameters (which allows us to test the quality of the computed coefficients) and, at the same time, the graph of the Weierstraß function is "strange" enough. This idea is borrowed from [dlLP02], but since we use more data than in [dlLP02] we reproduce the example.

Example 4.6. From Section 3 we know that $\mathfrak{W}_{A, B} \in \mathscr{B}_{\infty, \infty}^{-\log _{B}(A)}(\mathbb{R})$. To test the algorithm we fix the parameter $B=3$ and we take $A \in[0.4,0.8]$. Hence, $\mathfrak{W}_{A, 3} \in \mathscr{B}_{\infty, \infty}^{s}(\mathbb{R})$ with $s=-\log _{3}(A) \in[0.20311 \ldots, 0.83404 \ldots]$. Then, observe that

$$
1<\max \left(s, \frac{5}{2}-s\right)=\frac{5}{2}-s<3 .
$$

Therefore the above algorithm is valid in this case only for Daubechies wavelets with $k \geq 3$ vanishing moments.

To perform the above algorithm we take $J=30$ (that is, we use a sample of the graph of $\mathfrak{W}_{A, 3}$ of $2^{30}$ points). To carry out Step 1, by Lemma 2.3, we can estimate

$$
a_{-J}[n] \approx 2^{-J / 2} \mathfrak{W}_{A, 3}\left(2^{-J} n\right) .
$$

In Subsection 6.1 we will obtain the sample of the function that we want to study over a piece of an orbit of an irrational rotation $R_{\omega}$. So, to test the algorithm it is advisable to compute also the regularities of the Weierstraß function in the same conditions. More precisely, we obtain the sample $\left\{\mathfrak{W}_{A, 3}\left(\theta_{n}\right)\right\}_{n=0}^{2^{30}-1}$, where $\theta_{0} \in \mathbb{S}^{1}$ and $\theta_{n+1}:=R_{\omega}\left(\theta_{n}\right)$ for $n=0, \ldots, 2^{30}-1$.

Then, after executing Steps 1-4 of the above algorithm we obtain the results depicted in Figures 1-2.
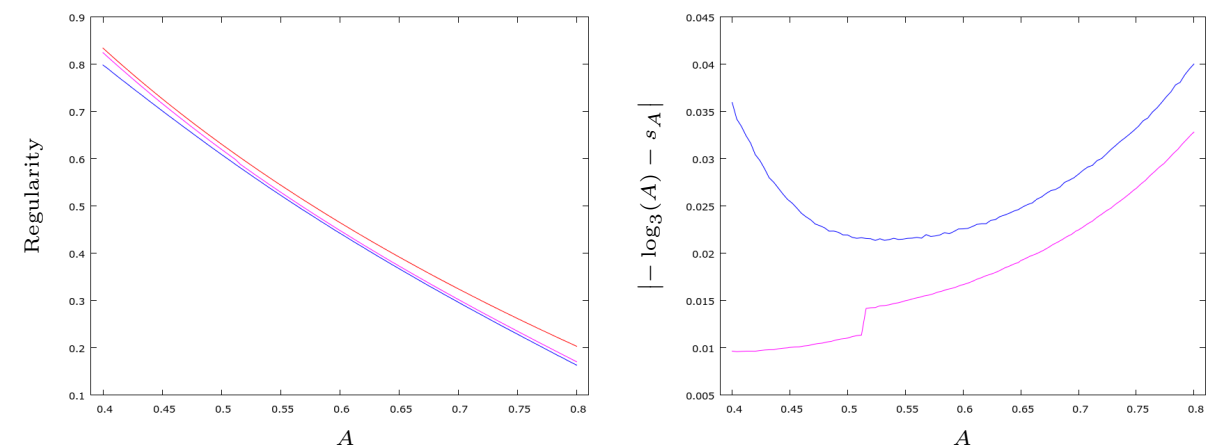

FiguRE 1. On the left picture the theoretical and estimated regularity of $\mathfrak{W}_{A, 3}$ with $A \in[0.4,0.8]$ are shown. The theoretical curve is plotted in red whereas the numerical ones are blue for the rotated version (computed with the D8 wavelet) and the non-rotated version of the Weierstraß function in magenta (computed with the D12 wavelet). On the right picture the error $\left|-\log _{3}(A)-s_{A}\right|$ is plotted (here $s_{A}$ denotes the estimated regularity of $\mathfrak{W}_{A, 3}$ ). 
We want to remark that the best numerical estimate of the regularity of $\mathfrak{W}_{A, 3}(x)$, with $A \in[0.4,0.8]$, it is computed with a Daubechies wavelet of 6 vanishing moments and the range of $j$ 's is $-29 \leq j<-4$. It turns out that D12 wavelet also maximizes globally the computed Pearson correlation coefficients in the range of parameters that we consider. Hence, despite of the inherited error of the FWT's seed, D12 is the best to approximate and to explain the regularity of the Weierstraß function (in the sense that minimizes the error). The same comments apply in the case of the rotated version of $\mathfrak{W}_{A, 3}(x)$, with $A \in[0.4,0.8]$. In this case the chosen wavelet is D8. The error of such estimates are represented on the right hand side of Figure 1.

We also want to remark that all the computed Pearson correlation coefficients are bigger than 0.999. This agrees with the fact that the Weierstraß function is self-similar. Then as pointed out in Remarks 4.3 and 4.5 , the coefficients $d_{j}[n]$ must be approximately on a straight line. However, in concordance with Remark 4.5, there are pairs $\left(j, s_{j}\right)$ which are not on a straight line and they are not taken into account in the regularity estimation. This is what Figure 2 shows for a certain cases of the Weierstraß function evaluated on the points $n / 2^{J}$. Finally, we want to say that the observed error of the estimated regularity is smaller than $5 \times 10^{-2}$. Also, the behaviour of the regularities of $\mathfrak{W}_{A, 3}(x)$ and $\mathfrak{W}_{A, 3}\left(R_{\omega}(x)\right)$, with the parameter $A \in[0.4,0.8]$ are similar.

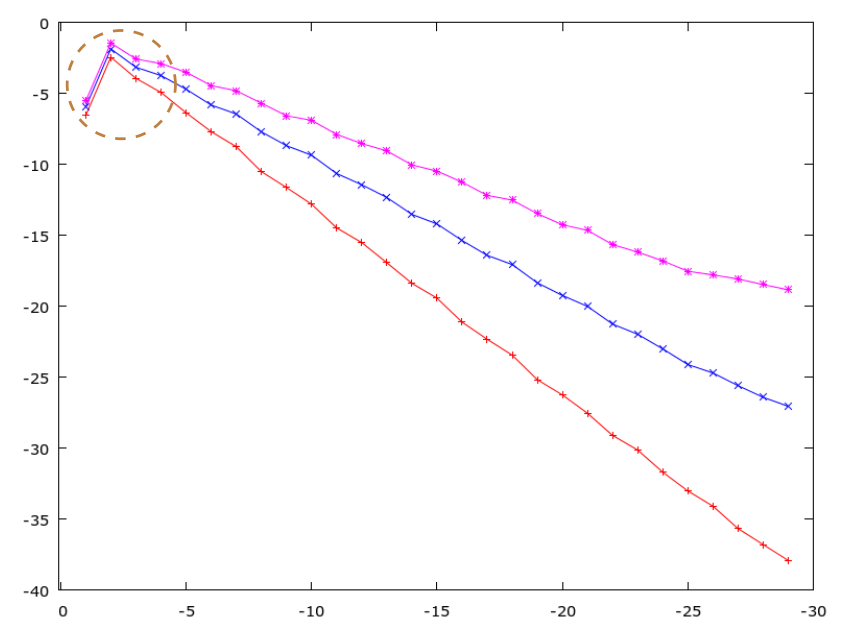

Figure 2. The connect the dot graphs of the pairs $\left(j, s_{j}\right)$ with $-29 \leq j \leq 0$ for $\mathfrak{W}_{0.4,3}$ in red, $\mathfrak{W}_{0.6,3}$ in blue and $\mathfrak{W}_{0.8,3}$ in magenta. The values inside the dashed circle are not used on the computations of the regularities for the reasons explained in Remark 4.5.

In view of the results obtained in Example 4.6, in the next sections we will use the same algorithm with a family of a Strange Non-Chaotic Attractors.

\section{AN UPPER SEMI-CONTINUOUS SNA}

In [GOPY84], a quasi-periodically forced skew product on the cylinder was studied. The attractor obtained there (as shown by Keller in [Kel96]), is the graph of an upper semi-continuous function. This kind of systems will be the test case for the algorithms that we are going to develop. 
We start by introducing the model (and the attractor) that we are going to study following [Kel96]. We consider skew products on the Cartesian product of the circle $\mathbb{S}^{1}=\mathbb{R} / \mathbb{Z}$ and $\mathbb{R}^{+}=[0, \infty)$ of the type

$$
\left(\begin{array}{c}
\theta_{k+1} \\
x_{k+1}
\end{array}\right)=\mathfrak{F}_{\sigma, \varepsilon}\left(\theta_{k}, x_{k}\right)=\left(\begin{array}{c}
R_{\omega}\left(\theta_{k}\right) \\
f_{\sigma}\left(x_{k}\right) g_{\varepsilon}\left(\theta_{k}\right)
\end{array}\right)
$$

where $\left(\theta_{k}, x_{k}\right) \in \mathbb{S}^{1} \times \mathbb{R}^{+}, R_{\omega}\left(\theta_{k}\right)=\theta_{k}+\omega(\bmod 1)$ and $\omega \in \mathbb{R} \backslash \mathbb{Q}$. On the second component, the map $g_{\varepsilon}: \mathbb{S}^{1} \longrightarrow[0, \infty)$ is continuous (hence bounded-for example $(\varepsilon+|\cos (2 \pi \theta)|))$ and the map $f_{\sigma}:[0, \infty) \longrightarrow[0, \infty)$ is $\mathcal{C}^{1}$, bounded, increasing, strictly concave and such that $f_{\sigma}(0)=0$ (e.g. $\left.\left.2 \sigma \tanh (x)\right|_{\mathbb{R}^{+}}\right)$. Observe that, since $f_{\sigma}(0)=0$, the circle $x \equiv 0$ is invariant.

Recall that the vertical Lyapunov Exponent at a point $\left(\theta_{0}, x_{0}\right)$ is defined by

$$
\limsup _{k \rightarrow \infty} \frac{1}{k} \log \left\|\left(\begin{array}{cc}
1 & 0 \\
\frac{\partial x_{k}}{\partial \theta} & \frac{\partial x_{k}}{\partial x}
\end{array}\right)\left(\begin{array}{l}
0 \\
1
\end{array}\right)\right\|=\underset{k \rightarrow \infty}{\limsup } \frac{1}{k} \log \left|\frac{\partial x_{k}}{\partial x}\right| .
$$

Therefore, by the Birkhoff Ergodic Theorem, it can be shown that the vertical Lyapunov Exponent at $x \equiv 0$ is

$$
\kappa\left(f_{\sigma}, g_{\varepsilon}\right):=\int_{\mathbb{S}^{1}} \log \left|\frac{\partial f_{\sigma}(x) g_{\varepsilon}(\theta)}{\partial x}\right|_{x=0}\left|d \theta=\log \left(f_{\sigma}^{\prime}(0)\right)+\int_{\mathbb{S}^{1}} \log \right| g_{\varepsilon}(\theta) \mid d \theta .
$$

When $\kappa\left(f_{\sigma}, g_{\varepsilon}\right)$ is positive, $x \equiv 0$ is a repellor for system (14). Moreover, since $f_{\sigma}$ and $g_{\varepsilon}$ are bounded, infinity is also a repellor and the system must have an attractor different from $x \equiv 0$. These attractors are typically very complicated.

We are going to restrict ourselves to the study of a particular subfamily of model (14) which is

$$
\left(\begin{array}{c}
\theta_{k+1} \\
x_{k+1}
\end{array}\right)=\mathfrak{F}_{\sigma, \varepsilon}\left(\theta_{k}, x_{k}\right)=\left(\begin{array}{c}
R_{\omega}\left(\theta_{k}\right) \\
2 \sigma \tanh \left(x_{k}\right) \cdot\left(\varepsilon+\left|\cos \left(2 \pi \theta_{k}\right)\right|\right)
\end{array}\right),
$$

with $\omega=\frac{1+\sqrt{5}}{2}, \sigma>0$ and $\varepsilon \geq 0$. Apart from the parameter $\varepsilon$, it is the natural restriction to $\mathbb{R}^{+}$of the system considered in [GOPY84] (see Figure 3, where a graph of the attractor of this system with $\sigma=1.5$ and $\varepsilon=0$ is shown). In this case, the vertical exponent $\kappa\left(f_{\sigma}, g_{\varepsilon}\right)$ at $x \equiv 0$ is precisely $\log (\sigma)$. Hence, the interesting case (for us) occurs when $\sigma>1$.

The attractor of system (14) and its dynamics are described by the following theorem (see also Figure 3 for an illustration of the graph of this attractor):

Theorem 5.1 (G. Keller, [Kel96]). There exists an upper semi-continuous function $\varphi: \mathbb{S}^{1} \longrightarrow \mathbb{R}^{+}$whose graph is invariant under system (14) and satisfies

(a) The Lebesgue measure on the circle, lifted to the graph of $\varphi$ is a Sinai-RuelleBowen measure (that is,

$$
\lim _{k \rightarrow \infty} \frac{1}{k} \sum_{i=0}^{k-1} f_{\sigma}\left(\mathfrak{F}_{\sigma, \varepsilon}^{i}(\theta, x)\right)=\int_{\mathbb{S}^{1}} f_{\sigma}(\theta, \varphi(\theta)) d \theta
$$

for every $f \in \mathcal{C}^{0}\left(\mathbb{S}^{1} \times \mathbb{R}^{+}, \mathbb{R}\right)$ and Lebesgue almost every $\left.(\theta, x) \in \mathbb{S}^{1} \times \mathbb{R}^{+}\right)$,

(b) if $\kappa\left(f_{\sigma}, g_{\varepsilon}\right) \leq 0$ then $\varphi \equiv 0$,

(c) if $\kappa\left(f_{\sigma}, g_{\varepsilon}\right)>0$ then $\varphi(\theta)>0$ for almost every $\theta$,

(d) if $\kappa\left(f_{\sigma}, g_{\varepsilon}\right)>0$ and $g_{\varepsilon}$ vanishes at some point then the set $\left\{\theta \in \mathbb{S}^{1}: \varphi(\theta)>0\right\}$ is meager and $\varphi$ is almost everywhere discontinuous,

(e) if $\kappa\left(f_{\sigma}, g_{\varepsilon}\right)>0$ and $g_{\varepsilon}>0$ then $\varphi$ is positive and continuous; if $g_{\varepsilon} \in \mathcal{C}^{1}$ then so is $\varphi$,

(f) if $\kappa\left(f_{\sigma}, g_{\varepsilon}\right) \neq 0$ then $\left|x_{n}-\varphi\left(\theta_{n}\right)\right| \rightarrow 0$ exponentially fast for almost every $\theta$ and every $x>0$. 


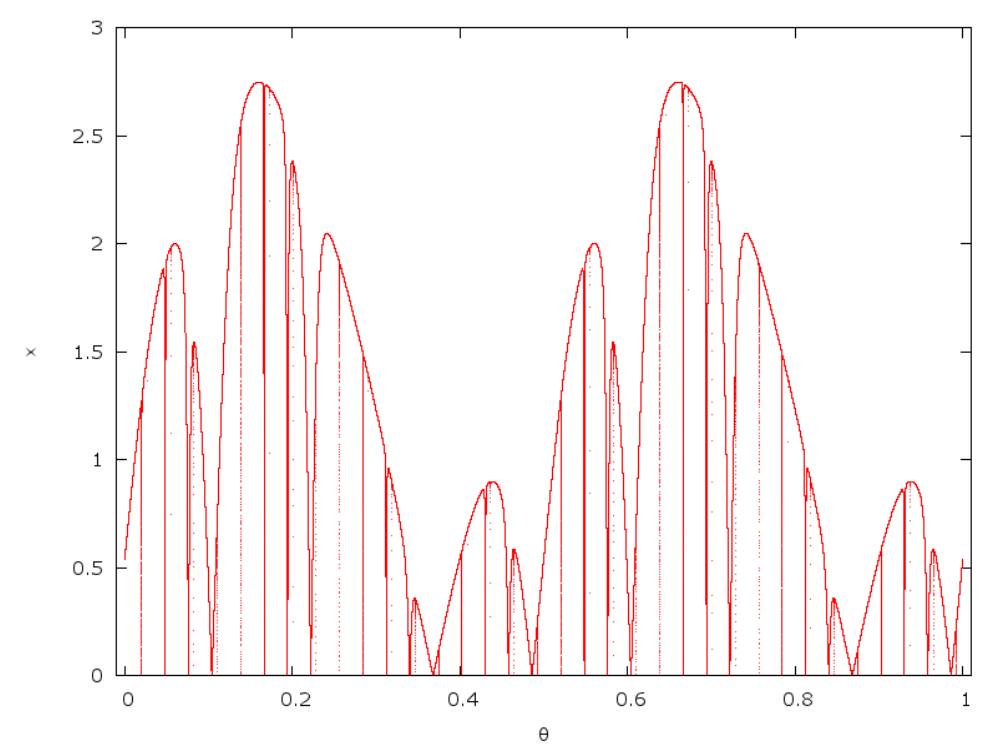

FiguRE 3. The attractor of system (15) for $\sigma=1.5$ and $\varepsilon=0$. Notice the abrupt changes in the graph of the attractor.

Observe that, when $\kappa\left(f_{\sigma}, g_{\varepsilon}\right)>0$ and $g$ vanishes at some point (i.e. there exists a fibre whose image is degenerate to a point), it follows from statements $(\mathrm{c}, \mathrm{d})$ that $\varphi$ is discontinuous almost everywhere. If there exists $\theta_{0} \in \mathbb{S}^{1}$ such that $g_{\varepsilon}\left(\theta_{0}\right)=0$, we will say that the system is pinched. In the particular case of system (15), the pinching condition implies that $\varepsilon=0$ and, since $|\cos (2 \pi \theta)|$ vanishes for $\theta \in\left\{\frac{1}{4}, \frac{3}{4}\right\}$, it follows that the set

$$
\left\{\left(\frac{i}{4}+k \omega \quad(\bmod 1), 0\right): k \in \mathbb{N}, i \in\{1,3\}\right\}
$$

is both a subset of the attractor and is dense (and invariant) in $x \equiv 0$. On the other hand, if $\varepsilon>0$ we can not have a dense set of pinched points.

The proof of the above theorem is based on the iteration of the Transfer Operator of the system and many of the properties of $\varphi$ can be derived from such iteration. Since we will strongly use this construction let us briefly explain it. Let $\mathscr{P}$ be the space of all functions (not necessarily continuous) from $\mathbb{S}^{1}$ to $\mathbb{R}$. If we look for a functional version of the system (14) in the space $\mathscr{P}$ one can define the Transfer Operator $\mathfrak{T}: \mathscr{P} \longrightarrow \mathscr{P}$ as

$$
\mathfrak{T}(\varphi)(\theta)=f_{\sigma}\left(\varphi\left(R_{\omega}^{-1}(\theta)\right)\right) \cdot g_{\varepsilon}\left(R_{\omega}^{-1}(\theta)\right) .
$$

Remark 5.2. From the above definition we obtain

$$
\mathfrak{T}(\varphi)(\theta)=\pi_{x}\left(\mathfrak{F}_{\sigma, \varepsilon}\left(R_{\omega}^{-1}(\theta), \varphi\left(R_{\omega}^{-1}(\theta)\right)\right)\right)
$$

where $\pi_{x}: \mathbb{S}^{1} \times \mathbb{R}^{+} \longrightarrow \mathbb{R}^{+}$denotes the projection with respect to the second component.

Notice that the graph of a function $\varphi: \mathbb{S}^{1} \longrightarrow \mathbb{R}$ is invariant for the system (14) if and only if $\mathfrak{T}(\varphi)=\varphi$.

To obtain the map $\varphi$ from Theorem 5.1, Keller takes a sufficiently large constant function $\varphi_{0}=c$ (with $c>\sup _{x \in \mathbb{R}} f_{\sigma}(x) \max _{\theta \in[0,1]} g_{\varepsilon}(\theta)$ ) and iterates it under the transfer operator $\mathfrak{T}$ (see Figure 4 ). In such a way he gets, since the map $f$ is monotone, a non-increasing sequence of continuous maps given by

$$
\varphi_{k}=\mathfrak{T}\left(\varphi_{k-1}\right)=\mathfrak{T}^{k}(c),
$$




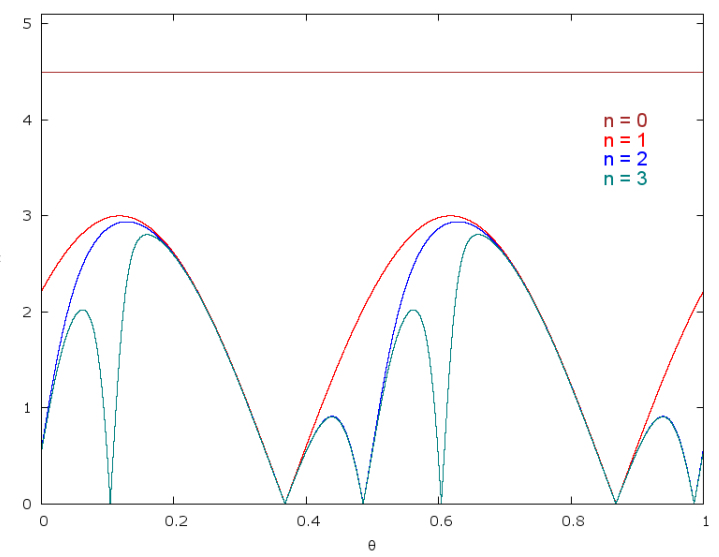

FiguRE 4. The constant function $c=5$ and three iterations of the Transfer Operator $\mathfrak{T}$ for system 15 with $\sigma=1.5$ and $\varepsilon=0$. The function $c$ is plotted in brown, $\mathfrak{T}(c)$ in red, $\mathfrak{T}^{2}(c)$ in blue and $\mathfrak{T}^{3}(c)$ in teal.

where $\mathfrak{T}^{k}$ stands for the $k$-th iterate of the Transfer Operator. Then, following Keller's proof, one has that

$$
\varphi:=\lim _{k \rightarrow \infty} \varphi_{k}=\inf _{k \rightarrow \infty} \varphi_{k}
$$

and the point-wise convergence of the above sequence is exponentially fast. Notice that the shape of $\varphi$ depends on the parameters $\sigma$ and $\varepsilon$.

Moreover, when proving the upper semi continuity of $\varphi$, it is shown that all sets $\left\{\theta \in \mathbb{S}^{1}: \varphi(\theta)<\varepsilon\right\}$ are open. This means that $\varphi$ is continuous at each point where $g$ vanishes (whether pinched or non-pinched) and, also, it is in $\mathscr{L}^{1}\left(\mathbb{S}^{1}\right)$. That is, the function $\varphi \in \mathscr{L}^{1}\left(\mathbb{S}^{1}\right)$ is continuous at

$$
\mathbf{Z}_{(n)}:=\left\{Z_{g_{\varepsilon}}+k w \quad(\bmod 1): k=0, \ldots, n\right\}=\bigcup_{k=0}^{n} R_{\omega}^{k}\left(Z_{g_{\varepsilon}}\right) \subset \mathbb{S}^{1}
$$

where $Z_{g_{\varepsilon}} \subset \mathbb{S}^{1}$ is the finite and discrete set where the function $g_{\varepsilon}$ vanishes.

5.1. On the regularity of the attractor. The regularity of the attractor of system (14) in terms of $\varepsilon$ and the regularity of $g_{\varepsilon}$ is given by the following

Proposition 5.3. Let $\varphi: \mathbb{S}^{1} \longrightarrow \mathbb{R}^{+}$be the upper semi-continuous function whose graph is invariant under system (14). Assume that $g_{\varepsilon} \in \mathscr{B}_{\infty, \infty}^{s}\left(\mathbb{S}^{1}\right)$ with $s \in(0,1]$.

(a) If $\varepsilon>0$ then $\varphi \in \mathscr{B}_{\infty, \infty}^{s}\left(\mathbb{S}^{1}\right)$.

(b) If $\varepsilon=0$ then $\varphi \in \mathscr{B}_{\infty, \infty}^{0}\left(\mathbb{S}^{1}\right)$.

Proof. Statement (a) follows from [Sta99, Theorem 1.2] and Statement (b) follows from Theorem 5.1 and Lemma 3.6.

Remark 5.4: The choice of a convenient $g_{\varepsilon}$ can lead us to a $\varphi$ with zero regularity (independently on whether the system is pinched or non-pinched). Assume that there is an $\alpha>0$ such that

$$
\lim _{\theta \rightarrow 0} \frac{|\theta|^{\alpha}}{g_{\varepsilon}(\theta)}=0
$$

(this means that $g_{\varepsilon}(\theta)$ does not verify any Hölder condition but it is continuous see Example 3.4(ii)). By Keller's Theorem, the invariant function $\varphi$ is continuous 
when $\varepsilon>0$. However, by the choice of $g_{\varepsilon}$, it cannot verify any Hölder condition. Therefore, $\varphi \in \mathscr{B}_{\infty, \infty}^{0}\left(\mathbb{S}^{1}\right)$.

\section{An algorithm to COMpute the Wavelet COefFicients AND REGULARITIES OF THE ATTRACTORS OF SYSTEM (14)}

We want to approximate the invariant curve $\varphi$ of the system (15) in terms of wavelets with quality control. Since $g_{\varepsilon}(\theta)=\varepsilon+|\cos (2 \pi \theta)|$ is Lipschitz then, by Proposition 5.3, we know that the regularity of the attractor is 0 when $\varepsilon=0$ and 1 when $\varepsilon>0$. The quality control (of the wavelet coefficients) is implemented by comparing this theoretical value with the estimate of the regularity obtained from the wavelet coefficients.

Since the attractor of system (15) is the graph of a map $\varphi: \mathbb{S}^{1} \longrightarrow \mathbb{R}^{+}$we will use the methodology described in the previous section applied to the function $\varphi^{\mathrm{PER}}$ (see Lemma 3.8 and Remark 4.4). However, $\varphi^{\mathrm{PER}}$, in the pinched case, is discontinuous almost everywhere (and the corresponding attractor is called strange). Therefore, we are not allowed to apply verbatim the algorithm from the previous section. In the rest of this section we will describe how to solve this problem in the implementation of the strategy from the previous section.

More concretely, to compute an approximation of the type (12) for $\varphi^{\mathrm{PER}}$, since we do not have an explicit formula for $\varphi$, we will use Theorem 5.1(f) and the transfer operator to get a sufficiently good numerical approximation of this function. Indeed, by Theorem 5.1 (f), for almost every $\theta_{0} \in \mathbb{S}^{1}$, any $x_{0}>0$ and any $\varepsilon>0$ there exists $N_{0}$ such that for every $n \geq N_{0}$ we have:

$$
\left|x_{n}-\varphi\left(\theta_{n}\right)\right|<\varepsilon
$$

where $\left(\theta_{n}, x_{n}\right)=\mathfrak{F}_{\sigma, \varepsilon}^{n}\left(\theta_{0}, x_{0}\right)$. Moreover, the points $\left(\theta_{n}, x_{n}\right)$ with $n \in\left\{N_{0}, N_{0}+\right.$ $\left.1, \ldots, N_{0}+2^{J}-1\right\}$ approximate exponentially fast the points $\left(\theta_{n}, \varphi\left(\theta_{n}\right)\right)$ from $\operatorname{graph}(\varphi)$. Therefore,

$$
\left\{\left(\theta_{n}, x_{n}\right): n=N_{0}, N_{0}+1, \ldots, N_{0}+2^{J}-1\right\}
$$

is an approximate mesh of $\operatorname{graph}(\varphi)$ provided that $J$ is large enough. To fix the mesh we choose a random point $\theta_{0}$ and we fix some $x_{0}>\sup _{x \in \mathbb{R}}+2 \sigma \tanh (x)=2 \sigma$. However, this approximate mesh has two problems to be used in our computations:

Problem (1) as we will see, we need a mesh of the graph of $\varphi$ at dyadic points of the form $i 2^{-J}$ for $i=0,1, \ldots, 2^{J}-1$,

Problem (2) we cannot use Lemma 2.3 to estimate the initial coefficients $a_{-J}[n]$ since our map $\varphi$ is discontinuous almost everywhere (and, hence, not Lipschitz).

In the following two subsections, we will explain how one can solve the above two problems.

6.1. A solution to Problem (1): a $\mathcal{C}^{2}$ homeomorphism. As we have said, we need a mesh of the graph of $\varphi$ at dyadic points of the form $\theta_{i}=i 2^{-J}$ for $i=0,1, \ldots, 2^{J}-1$ but, clearly, if we obtain the points $\left(\theta_{n}, x_{n}\right)$ just as iterates of a single point by $\mathfrak{F}_{\sigma, \varepsilon}$ this condition is not satisfied. The natural approach which would be to approximately compute the points of the graph of $\varphi$ based at the dyadic points by interpolating the obtained values is not feasible since, by Theorem 5.1 , we know that $\varphi$ is upper semi-continuous and discontinuous everywhere. Then we propose the following solution which consists in moving to a conjugate system with the desired properties. To do this, first we relabel the points $\left\{\left(\theta_{n}, x_{n}\right)\right\}_{n=N_{0}}^{N_{0}+2^{J}-1}$ to a sequence $\left\{\left(\widetilde{\theta}_{i}, z_{i}\right)\right\}_{i=0}^{2^{J}-1}$ so that

$$
0 \leq \widetilde{\theta}_{0}<\widetilde{\theta}_{1}<\cdots<\widetilde{\theta}_{2^{J}-1}<1
$$


(we do this simply by sorting the data (18) with respect to the first coordinate; see Remark 6.4). In particular if $n \in\left\{N_{0}, N_{0}+1, \ldots, N_{0}+2^{J}-1\right\}$ and $i=i(n) \in$ $\left\{0,1, \ldots, 2^{J}-1\right\}$ is such that $\widetilde{\theta}_{i}=\theta_{n}$, then $z_{i}=x_{n}$.

Definition 6.1. Let $h: \mathbb{S}^{1} \longrightarrow \mathbb{S}^{1}$ be the unique $\mathcal{C}^{2}$-monotone rational spline of degree one with nodes $\left(\frac{i}{2^{J}}, \widetilde{\theta}_{i}\right)$ for $i=0, \ldots, 2^{J}-1$ and $\left(1, \widetilde{\theta}_{0}+1\right)$. In particular $h(1)=\widetilde{\theta}_{0}+1$ and $h\left(\frac{i}{2^{J}}\right)=\widetilde{\theta}_{i}$ for $i=0, \ldots, 2^{J}-1$. We also define the map

$$
\varphi \circ h: \mathbb{S}^{1} \longrightarrow \mathbb{R}^{+}
$$

Clearly $\left\{\left(i 2^{-J}, z_{i}\right)\right\}_{i=0}^{2^{J}-1}$ is now an approximate mesh of $\operatorname{graph}(\varphi \circ h)$ which is based at the dyadic points. Thus, we will use the list of pairs $\left\{\left(i 2^{-J}, z_{i}\right)\right\}_{i=0}^{2^{J}-1}$ to estimate the regularity of $\varphi \circ h$. In the Appendix, following [Del83], we will construct the $\mathcal{C}^{2}$ homeomorphism $h$ and show that

$$
\|h\|_{\mathcal{C}^{1}} \leq 7(1+\sqrt{5}) \text {. }
$$

Remark 6.2. The map $\varphi \circ h$ has the following dynamical interpretation. Consider the homeomorphism $H: \mathbb{S}^{1} \times \mathbb{R}^{+} \longrightarrow \mathbb{S}^{1} \times \mathbb{R}^{+}$defined by $H(\theta, x)=(h(\theta), x)$. Then, using similar ideas as the ones in [Kel96] it can be shown that, $\operatorname{graph}(\varphi \circ h)$ is the attractor of the dynamical system

$$
\left(H^{-1} \circ \mathfrak{F}_{\sigma, \varepsilon} \circ H\right)(\theta, x)=\left(h^{-1}\left(R_{\omega}(h(\theta))\right), f_{\sigma}(x) g_{\varepsilon}(h(\theta))\right),
$$

which is conjugate to system (15). Indeed, by using (with minor changes) the proof of the Statement 1 from [Kel96, Theorem 1] one can see that $(\varphi \circ h)(\theta)$ is the pointwise limit of a non-increasing sequence of continuous functions. In other words, $(\varphi \circ h)(\theta)=\lim _{k \rightarrow \infty} \varphi_{k}(h(\theta))$ for every $\theta$. Therefore $\operatorname{graph}(\varphi \circ h)$ is the attractor of the system (20) which is conjugate, by $H$, to the one given by (14) (and hence to $(15))$.

The next result (c.f. [Tri92, Section 4.3]) tells us that, in this situation the regularity of $\varphi$ coincides with the regularity of $\varphi \circ h$ and, hence, we can estimate them by using the mesh $\left\{\left(i 2^{-J}, z_{i}\right)\right\}_{i=0}^{2^{J}-1}$.

Proposition 6.3. Let $f \in \mathscr{B}_{\infty, \infty}^{s}\left(\mathbb{S}^{1}\right)$ with $s \in \mathbb{R}$ and let $h: \mathbb{S}^{1} \longrightarrow \mathbb{S}^{1}$ be a $\mathcal{C}^{m}$ diffeomorphism with $m \geq s$. Then $f \circ h$ belongs to $\mathscr{B}_{\infty, \infty}^{s}\left(\mathbb{S}^{1}\right)$.

We remark that the exact formula for $h$ is irrelevant for our algorithm. We only use its properties. That is, we only take into account the fact that such a homeomorphism $h$ exists and that it is $\mathcal{C}^{2}$. In other words, we elude the need to effectively interpolate. Indeed, to obtain the data mesh $\left\{\left(i 2^{-J}, z_{i}\right)\right\}_{i=0}^{2^{J}-1}$ that approximates $\operatorname{graph}(\varphi \circ h)$ (the attractor of the conjugate system) we simply have to sort the computed mesh $\left\{\left(\theta_{n}, x_{n}\right)\right\}_{n=N_{0}}^{N_{0}+2^{J}-1}$ of $\operatorname{graph}(\varphi)$ with respect to the first coordinate $\theta$ to obtain $\left\{\left(\widetilde{\theta}_{i}, z_{i}\right)\right\}_{i=0}^{2^{J}-1}$ and delete the first mesh components $\widetilde{\theta}_{i}$ (thus implicitly assuming that the new mesh is $\left.\left\{\left(i 2^{-J}, z_{i}\right)\right\}_{i=0}^{2^{J}-1}\right)$. Of course, this does not add any further computational error to the mesh. Furthermore, as it has been already said, the exponential contraction of the system to the attractor (see Theorem 5.1(f)) still holds for the conjugate system, thus assuring that there is no loss of precision when replacing $\varphi$ by $\varphi \circ h$ and $\left\{\left(\theta_{n}, x_{n}\right)\right\}_{n=N_{0}}^{N_{0}+2^{J}-1}$ by $\left\{z_{i}\right\}_{i=0}^{2^{J}-1}$ (see Subsection 6.2). However, there is a kind of chain rule in Proposition 6.3. Indeed, if $C(\varphi)$ denotes the constant $C$ from Theorem 4.2, then equation (19) gives

$$
C(\varphi \circ h) \approx C(\varphi)\|h\|_{\mathcal{C}^{1}} \leq 7(1+\sqrt{5}) C(\varphi) .
$$


That is, the effect of working with $\varphi \circ h$ instead of $\varphi$, as it can be clearly deduced from Figure 1, is to change the constant term of the linear regression model (13) in a way independent on $J$ (and $N$ ).

Finally, we note that the proposed method works because an irrational rotation $R_{\omega}$ is uniquely ergodic and, hence, its unique invariant probability measure is the Lebesgue measure on $\mathbb{S}^{1}$, and the base map of system $(20)$, being $\mathcal{C}^{2}$-conjugate to an irrational rotation, has an invariant measure which is absolutely continuous with respect to the Lebesgue measure.

Remark 6.4. The process of sorting the data of an array of $2^{30}$ points from $\mathbb{S}^{1} \times \mathbb{R}^{+}$ (stored as pairs of double variables in C) turns to be the bottleneck of the whole algorithm (and the most time consuming task of the whole program). Moreover, even the process of computing and filling the array with the initial mesh of the function $\varphi$ already spends a "visible" amount of CPU time. Indeed, the iteration, storing and sorting process (with a standard sort algorithm like Heapsort) of this data spends about $2200 \mathrm{CPU}$ seconds, with a remarkable variability which depends on the initial sorting of the data, in a computer with a Xeon processor at $3 \mathrm{GHz}$ and $32 \mathrm{~Gb}$ of RAM memory. In order to reduce the time elapsed in the sorting process we use the following trick based on the fact that the dynamical system generating the $\theta_{i}$ data is the irrational rotation $R_{\omega}$. In this case we know that the Lebesgue measure is the unique ergodic measure of $R_{\omega}$ and, hence, its averaged spatial distribution is uniform and it is controlled approximately by the Birkhoff's Ergodic Theorem applied to the Lebesgue measure. Indeed, we have

$$
\sharp\left(\left\{\theta, R_{\omega}(\theta), \ldots, R_{\omega}^{k-1}(\theta)\right\} \cap\left[\frac{i}{N}, \frac{i+1}{N}\right)\right) \approx \frac{k}{N}
$$

for $k$ large enough and for every $i \in\{0,1, \ldots, N-1\}$. The interpretation of this equation is that the statement

$$
\sharp\left(\left\{\theta_{N_{0}}, \theta_{N_{0}+1}, \ldots, \theta_{N_{0}+2^{J}-1}\right\} \cap\left[\frac{i}{2^{J}}, \frac{i+1}{2^{J}}\right)\right)=1
$$

holds with high frequency for $J$ large enough (observe that in this case we have $\left.\left\{\theta_{N_{0}}, \theta_{N_{0}+1}, \ldots, \theta_{N_{0}+2^{J}-1}\right\}=\left\{\theta_{N_{0}}, R_{\omega}\left(\theta_{N_{0}}\right), \ldots, R_{\omega}^{2^{J}-1}\left(\theta_{N_{0}}\right)\right\}\right)$. Moreover, when (21) holds, we have $i=\left\lfloor 2^{J} \theta_{l}\right\rfloor$, where $\theta_{l}$ is the unique element from the set $\left\{\theta_{N_{0}}, \theta_{N_{0}+1}, \ldots, \theta_{N_{0}+2^{J}-1}\right\} \cap\left[\frac{i}{2^{J}}, \frac{i+1}{2^{J}}\right)$ and $\lfloor\cdot\rfloor$ denotes the integer part function. This observation gives a good "hash function" and the following efficient algorithm to store and sort the data $\left\{\left(\theta_{n}, x_{n}\right)\right\}_{n=N_{0}}^{N_{0}+2^{J}}-1$. First, for $n=N_{0}, N_{0}+1, \ldots N_{0}+2^{J}-1$ we compute the point $\left(\theta_{n}, x_{n}\right)=\mathfrak{F}_{\sigma, \varepsilon}\left(\theta_{n-1}, x_{n-1}\right)$. Then, we store it in the position $i=\left\lfloor 2^{J} \theta_{n}\right\rfloor$ of the array data, if this slot is free. Otherwise, we store the point $\left(\theta_{n}, x_{n}\right)$ in a free position $j=j(i)$ of the array data such that $|j-i|$ is minimal. According to the above observations this will happen with low frequency and the array data will be almost sorted. Moreover, the positions of the array data which are not sorted are close to the place where they should be when the array is sorted. This is exactly the situation where the direct insertion sorting algorithm can be used with very good results. This means that we are using a method of order $\mathcal{O}\left(2^{J}+d\right)$ where $d$ is the number of insertions (which are very low due to the way we have stored all data) instead of a method of order $\mathcal{O}\left(J 2^{J}\right)$ as the Heapsort algorithm.

With this trick, the iteration, storing and sorting process lasts about $300 \mathrm{CPU}$ seconds, almost without variability, which clearly improves the efficiency of the program.

6.2. A solution to Problem (2): calculating the coefficients $a_{-J}^{\mathrm{PER}}[n]$ of $(\varphi \circ h)^{\mathrm{PER}}$. We introduce the following notation for the wavelet coefficients of the 
$\operatorname{map}(\varphi \circ h)^{\mathrm{PER}}$

$$
a_{j}^{\mathrm{PER}}[n]:=\left\langle(\varphi \circ h)^{\mathrm{PER}}, \phi_{j, n}\right\rangle \quad \text { and } \quad d_{j}^{\mathrm{PER}}[n]:=\left\langle(\varphi \circ h)^{\mathrm{PER}}, \psi_{j, n}\right\rangle
$$

for $j, n \in \mathbb{Z}$. When $\varphi \circ h$ is regular enough, Lemma 2.3 gives $2^{-J / 2}(\varphi \circ h)\left(\frac{n}{2^{J}}\right)$ as an estimate for the coefficients $a_{-J}^{\mathrm{PER}}[n]$. But, as we have pointed out, $\varphi$ (and hence $\left.(\varphi \circ h)^{\mathrm{PER}}\right)$ is discontinuous almost everywhere and the above estimate of $a_{-J}^{\mathrm{PER}}[n]$ is, a priori, not valid. However, as we will see, the element $z_{n} \approx(\varphi \circ h)\left(n 2^{-J}\right)$ from our data give indeed a good estimate for $a_{-J}^{\mathrm{PER}}[n]$ because our mesh is based at the dyadic points $n 2^{-J}$.

As it has been said in Section $5, \varphi$ is the point-wise limit of a non-increasing sequence of continuous (and, hence, uniformly continuous) functions $\varphi_{k}: \mathbb{S}^{1} \longrightarrow \mathbb{R}^{+}$ defined by

$$
\varphi_{0}(\theta)=c \quad \text { and } \quad \varphi_{k+1}(\theta)=\mathfrak{T}\left(\varphi_{k}\right)(\theta)
$$

for every $\theta \in \mathbb{S}^{1}$ and $c>\sup _{x \in \mathbb{R}^{+}} 2 \sigma \tanh (x)=2 \sigma$. Hence, recalling Remark 6.2, $(\varphi \circ h)(\theta)=\lim _{k \rightarrow \infty} \varphi_{k}(h(\theta))$ for every $\theta$; that is, $\varphi \circ h$ is the point-wise limit of a non-increasing sequence of continuous functions.

Remark 6.5. If we take $x_{0}=c=\varphi_{0}\left(\theta_{0}\right)$ then $x_{k}=\varphi_{k}\left(\theta_{k}\right)$ for every $k \geq 1$. To see this notice that, from the definition of the points $\left(\theta_{n}, x_{n}\right)$ and $\mathfrak{F}_{\sigma, \varepsilon}$, we get

$$
\theta_{k}=R_{\omega}\left(\theta_{k-1}\right) \quad \text { and } \quad x_{k}=\pi_{x}\left(\mathfrak{F}_{\sigma, \varepsilon}\left(\theta_{k-1}, x_{k-1}\right)\right)
$$

for every $k \geq 1$. Now, we proceed by induction. We assume that $x_{k-1}=\varphi_{k-1}\left(\theta_{k-1}\right)$ fore some $k \geq 0$. Then, by Remark 5.2 ,

$$
\begin{aligned}
x_{k} & =\pi_{x}\left(\mathfrak{F}_{\sigma, \varepsilon}\left(\theta_{k-1}, x_{k-1}\right)\right)=\pi_{x}\left(\mathfrak{F}_{\sigma, \varepsilon}\left(\theta_{k-1}, \varphi_{k-1}\left(\theta_{k-1}\right)\right)\right) \\
& =\mathfrak{T}\left(\varphi_{k-1}\right)\left(R_{\omega}\left(\theta_{k-1}\right)\right)=\varphi_{k}\left(\theta_{k}\right) .
\end{aligned}
$$

Since the scaling function $\phi$ of a Daubechies wavelet is continuous, so is $\phi_{-J, n}$ for each $n$. Hence, from the definition of the coefficients $a_{-J}^{\mathrm{PER}}[n]$ and the Dominated Convergence Theorem we have:

$$
\begin{aligned}
a_{-J}^{\mathrm{PER}}[n] & =\int_{\operatorname{supp}\left(\phi_{-J, n}\right)}(\varphi \circ h)^{\mathrm{PER}}(\theta) \phi_{-J, n}(\theta) d \theta \\
& =\lim _{k \rightarrow \infty} \int_{\operatorname{supp}\left(\phi_{-J, n}\right)}\left(\varphi_{k} \circ h\right)^{\mathrm{PER}}(\theta) \phi_{-J, n}(\theta) d \theta \\
& =\lim _{k \rightarrow \infty} a_{-J}^{k, \mathrm{PER}}[n],
\end{aligned}
$$

where $a_{-J}^{k, \mathrm{PER}}[n]:=\left\langle\left(\varphi_{k} \circ h\right)^{\mathrm{PER}}, \phi_{-J, n}\right\rangle$. From the proof of the Dominated Convergence Theorem, it can be shown that $a_{-J}^{k, \mathrm{PER}}[n]$ converge exponentially fast to $a_{-J}^{\mathrm{PER}}[n]$. Therefore, if $k$ is large enough, by Lemma 2.3 we have

$$
a_{-J}^{\mathrm{PER}}[n] \sim a_{-J}^{k, \mathrm{PER}}[n] \approx 2^{-J / 2}\left(\varphi_{k} \circ h\right)^{\mathrm{PER}}\left(n 2^{-J}\right)=2^{-J / 2} \varphi_{k}\left(h\left(n 2^{-J}\right)\right)
$$

for $n=0, \ldots, 2^{J}-1$ (where $\sim$ means exponentially close).

From the definition of $h$ it follows that, given $n \in\left\{0,1, \ldots, 2^{J}-1\right\}$, there exists $k \in\left\{N_{0}, N_{0}+1, \ldots, N_{0}+2^{J}-1\right\}$ such that $h\left(n 2^{-J}\right)=\widetilde{\theta}_{n}=\theta_{k}$. Therefore, by Remark 6.5,

$$
\varphi_{k}\left(h\left(n 2^{-J}\right)\right)=\varphi_{k}\left(\theta_{k}\right)=x_{k}=z_{n} .
$$

Hence, if $N_{0}$ is large enough,

$$
a_{-J}^{\mathrm{PER}}[n] \approx 2^{-J / 2} \varphi_{k}\left(h\left(n 2^{-J}\right)\right)=2^{-J / 2} z_{n}
$$

for $n=0, \ldots, 2^{J}-1$. This gives the necessary approximation of the coefficients $a_{-J}^{\mathrm{PER}}[n]$ to initialize the algorithm. 
6.3. The algorithm for the system (14). In view of the previous sections and Section 4.1, we present the algorithm to estimate regularities, in terms of the Besov spaces $\mathscr{B}_{\infty, \infty}^{s}$, for the system (14).

Algorithm 6.6. Let $\mathfrak{F}_{\sigma, \varepsilon}$ be a skew product under the assumptions of the system (14). Fix $J>0$ and a transient $N_{0}>0$ big enough. To estimate the regularity of the invariant function, $\varphi$, of the system (14) perform the following steps:

Step 1. Generation of a mesh of points exponentially close to $\varphi$. By Theorem 5.1(f), fix $\sigma>1$, choose a random $\theta_{0} \in[0,1)$ and $x_{0}>1$ and, by using the recurrence $\left(\theta_{n}, x_{n}\right)=\mathfrak{F}_{\sigma, \varepsilon}\left(\theta_{n-1}, x_{n-1}\right)$, generate the data

$$
\left\{\left(\theta_{n}, x_{n}\right): n=N_{0}, N_{0}+1, \ldots, N_{0}+2^{J}-1\right\} \text {. }
$$

Step 2. Sort the data. Using Remark 6.4, sort the above data to obtain a sequence $\left\{\left(\widetilde{\theta}_{n}, z_{n}\right)\right\}_{n=0}^{2^{J}-1}$ so that

$$
0 \leq \widetilde{\theta}_{0}<\widetilde{\theta}_{1}<\cdots<\widetilde{\theta}_{2^{J}-1}<1,
$$

and delete the concrete values of the points $\widetilde{\theta}_{n}$. This defines a map $\varphi \circ h$, using Remark 6.2 and Proposition 6.3, with the same regularity as the map $\varphi$ such that $(\varphi \circ h)\left(n 2^{-J}\right) \approx z_{n}$ for $n=0, \ldots, 2^{J}-1$.

Step 3. FWT's initialization. Fix a Daubechies wavelet with $k$ vanishing moments and set $a_{-J}^{\mathrm{PER}}[n]:=2^{-J / 2} z_{n}$ for $n=0, \ldots, 2^{J}-1$. By equation (22) this is a good approximation of the coefficients $a_{-J}^{\mathrm{PER}}[n]$.

Now, since we have an approximation of $a_{-J}^{\text {PER }}[n]$, Steps $2-5$ of the strategy performed on Section 4.1 remain unaltered. That is,

Step 4. FWT procedure. Use equation (7) to calculate the wavelet coefficients $d_{-j}^{\mathrm{PER}}[n]=\left\langle f^{\mathrm{PER}}, \psi_{-j, n}\right\rangle$ for $j=0, \ldots, J-1$ and $0 \leq n \leq 2^{j}-1$.

Step 5. Application of Theorem 4.2: data to compute the regularity. By using the coefficients $d_{-j}^{\mathrm{PER}}[n]$ from Step 4 , calculate

$$
s_{-j}=\log _{2}\left(\sup _{0 \leq n \leq 2^{j}-1}\left|d_{-j}^{\mathrm{PER}}[n]\right|\right)
$$

for $j=0, \ldots, J-1$.

Step 6. Application of Remark 4.3 and 4.5: compute the regularity. Make a linear regression to estimate the slope $\tau$ of the graph of the pairs $\left(-j, s_{-j}\right)$ with $j=0, \ldots, J-1$ and recall that

$$
\mathrm{R}(t)= \begin{cases}t-\frac{1}{2} & \text { if } t>\frac{1}{2} \\ t+\frac{1}{2} & \text { if } t<-\frac{1}{2} \\ 0 & \text { if } t \in\left[-\frac{1}{2}, \frac{1}{2}\right] .\end{cases}
$$

Then, when there is evidence of linear correlation between the variables $-j$ and $s_{-j}$, we set $s=\mathrm{R}(\tau)$.

Step 7. Final test of assumptions of Theorem 4.2 on the number of vanishing moments. If $k>\max (s, 5 / 2-s)$ then $f \in \mathscr{B}_{\infty, \infty}^{s}(\mathbb{R})$ and, hence, $f$ has regularity $s$. Otherwise we need to repeat Step $4-7$ with a Daubechies wavelet having a larger value of $k$ until $k>\max (s, 5 / 2-s)$.

Remark 6.7. We want to emphasize that the above algorithm can be performed for other systems. The reason is that the "regularity steps", Steps 4-7, are valid for $f \in \mathscr{L}^{\infty}\left(\mathbb{S}^{1}\right)$ due to Theorem 4.2. Also notice that Steps $\mathbf{1}-\mathbf{3}$ can be skipped if the wavelet coefficients are obtained using other methods such as solving the Invariance 
Equation for example. Indeed, recall that the graph of a function $\varphi: \mathbb{S}^{1} \longrightarrow \mathbb{R}$ is invariant for the system (14) if and only if $\varphi$ is a fixed point of $\mathfrak{T}$. That is:

$$
f_{\sigma}\left(\varphi\left(R_{\omega}^{-1}(\theta)\right) \cdot g_{\varepsilon}\left(R_{\omega}^{-1}(\theta)\right)=\mathfrak{T}(\varphi)(\theta)=\varphi(\theta) .\right.
$$

The above equation is called the Invariance Equation. To give an approximation of $\varphi$ on a certain mesh of points $\theta_{i} \in \mathbb{S}^{1}$, one can solve a non-linear system of equations by imposing

$$
\varphi\left(\theta_{i}\right)=a_{0}+\sum_{j=0}^{J} \sum_{n=0}^{N_{j}} d_{j, n} \psi_{j, n}\left(\theta_{i}\right)
$$

on the Invariance Equation. The unknowns, like in the Fast Wavelet Transform, are the wavelet coefficients.

As a result, we can get an estimate of the regularity of the (strange) attractor of system (15) for the chosen value of $\sigma$ and $\varepsilon$. This will be the main topic of the following section.

\section{RESUlts AND CONCLUSIONS}

We have performed an exercise which is divided in two steps. The test case will be the system (15). The range of values for $\sigma$ is $[1,2]$ whereas $\varepsilon$ is given by the function

$$
\varepsilon(\sigma)= \begin{cases}(\sigma-1.5)^{2} & \text { when } 1.5<\sigma \leq 2 \\ 0 & \text { when } 1 \leq \sigma \leq 1.5\end{cases}
$$

That is, we will use the system

$$
\left(\begin{array}{l}
\theta_{k+1} \\
x_{k+1}
\end{array}\right)=\mathfrak{F}_{\sigma, \varepsilon(\sigma)}\left(\theta_{k}, x_{k}\right)=\left(\begin{array}{c}
R_{\omega}\left(\theta_{k}\right) \\
2 \sigma \tanh \left(x_{k}\right) \cdot\left(\varepsilon(\sigma)+\left|\cos \left(2 \pi \theta_{k}\right)\right|\right)
\end{array}\right) .
$$

Notice that with this parametrization, the above system is pinched if and only if $\sigma \in[1,1.5]$. Roughly speaking, the parameters $(\sigma, \varepsilon(\sigma))$ control the vertical Lyapunov Exponent of $x \equiv 0$ and the pinching condition at the same time. Hence, in view of Proposition 5.3 we can test the quality of the wavelet coefficients given by Algorithm 6.6. Indeed, we know that for $\sigma \in[1.5,2.0]$ the estimated value of $s$ must be close to 1 ; whereas for $\sigma \in[1.0,1.5]$ the regularity parameter $s$ must be zero. Moreover, when $\sigma$ crosses 1.5, in a decreasing way, the regularity parameter $s$ has to jump from 1 to 0 . Hence, the results obtained by Algorithm 6.6 applied to our problem and the fact that we can observe the jump of $s$ at $\sigma=1.5$ certify the quality of the algorithm that we have developed. Hence, we can have a degree of accuracy of the wavelet coefficients.

To do this test, first we have applied Algorithm 6.6 with $N_{0}=10^{5}$ and $J=30$. In Figure 5 we plot the estimated regularities of system (15) as a function of $\sigma$ with the above parametrization. Notice that, the estimated regularity detects the jump at $\sigma=1.5$ in a correct way and agrees with the values described above despite of the fact that the concrete values of the regularity are not correct for $\sigma \gtrsim 1.5$.

The choice of the Daubechies wavelet that we will use must be done carefully. Indeed, recall that Daubechies wavelets must have more than $\max (s, 5 / 2-s)$ vanishing moments in order to be under the assumptions of Theorem 4.2. However, the increase of the number of vanishing moments, $k$, causes an increment of the support size of the wavelet (see [Mal98, Theorem 7.3]). On the other hand, the support of the map $\varphi$ is $\mathbb{S}^{1}=\mathbb{R} / \mathbb{Z}=[0,1)$. Observe that, the ratio of growth between the support of $\varphi$ and $\psi_{-j, n}$ is 1 to $\frac{2 k-1}{2^{j}}$. Therefore, there exists $j_{0}$ such that for $j>j_{0}$ the support of $\psi_{-j, n}$ is contained in $[0,1)$. In view of that, the first coefficients of the wavelet approximation will be affected by an error induced by the size of the support of $\psi$. To avoid such bad performance, a good strategy is to choose different 

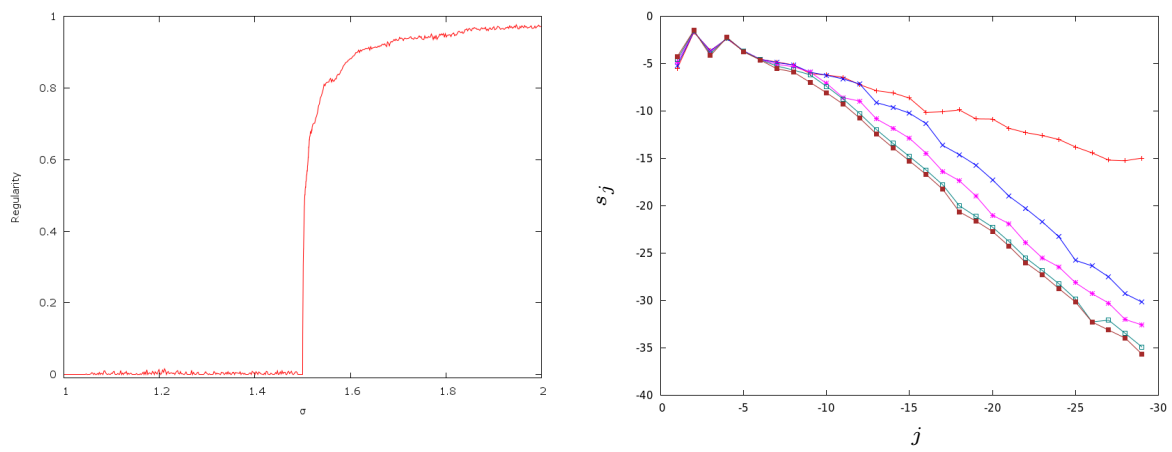

Figure 5. On the left picture one can see the estimate of the regularity $\mathrm{R}(\tau)$ of the (strange) attractor of system (15) for $\sigma \in$ $[1,2]$ and $\varepsilon$ given by the parametrization $\varepsilon(\sigma)$. The results are obtained by using a sample of $2^{30}$ points (that is, $J=30$ ), a transient $N_{0}=10^{5}$ and the wavelet D16. For this number of vanishing moments we obtain the minimum variance of Pearson correlation coefficient. On the right picture, in order to see how the slope of the linear model varies, there are the graphs of the pairs $\left(j, s_{j}\right)$ with $-29 \leq j \leq 0$ for $\sigma=1.49$ in red, $\sigma=1.51$ in blue, $\sigma=1.53$ in magenta, $\sigma=1.55$ in teal and $\sigma=1.57$ in brown.

wavelets for different parameters as it can be guessed in Figure 6. Having said that, Figure 5 (and its forthcoming discussion) is done with Daubechies wavelet with 8 vanishing moments: D16. This choice is based in two reasons. The first one is that all Daubechies wavelets used generate a picture similar as Figure 5. On the other hand, such wavelet explains better all the range of $\varepsilon$ (even the close to zero case as it can be seen in Figure 6). Additionally, for definiteness, we will exhibit the range of $j$ 's used in the numerical estimation of the regularity of $\varphi$ for the different values of $\varepsilon$ (see Remark 4.5).

Remark 7.1. Figure 6 has another interpretation. Indeed, the Daubechies wavelets with 7 and 8 vanishing moments explain (in terms of equation (13)) almost all the range of values of $\varepsilon$. However, there are regions of $\varepsilon$ where other wavelets are better than these ones. This means that a good strategy to perform Algorithm 6.6 in a more accurate way is to have a dictionary of wavelets. Notice that such dictionary cannot exists in the Fourier setting.

The second step of the exercise, and in view of the results displayed in Figure 5, is the explanation of the three regions that appear. Indeed, in Figure 5 one can clearly appreciate three regions with different qualitative behaviour. One of them corresponds to the pinched case (i.e. $\sigma \in[1,1.5]$ ) and the other two to the nonpinched one: $\sigma \in(1.5, \widetilde{\sigma})$ and $\sigma \in[\widetilde{\sigma}, 2]$ with $\widetilde{\sigma} \approx 1.527$. In what follows we discuss in detail these three regions.

Non pinched case: $\sigma \in[\widetilde{\sigma}, 2]$. In this region we have $\varepsilon=(\sigma-1.5)^{2} \gtrsim 7.29 \times$ $10^{-4}$. That is, we are "far" from the pinched case. As we already know, see Proposition 5.3, the function $\varphi$ whose graph is the attractor is continuous but not differentiable. Moreover, since we are far from the pinched case, $\varphi$ is rather well behaved since we have lack of differentiability only in few points (see the left picture of Figure 7). Precisely, the wavelet coefficients whose support contains these points should be large (of order $\simeq 2^{-j}$ ). This is confirmed by the estimated regularities 


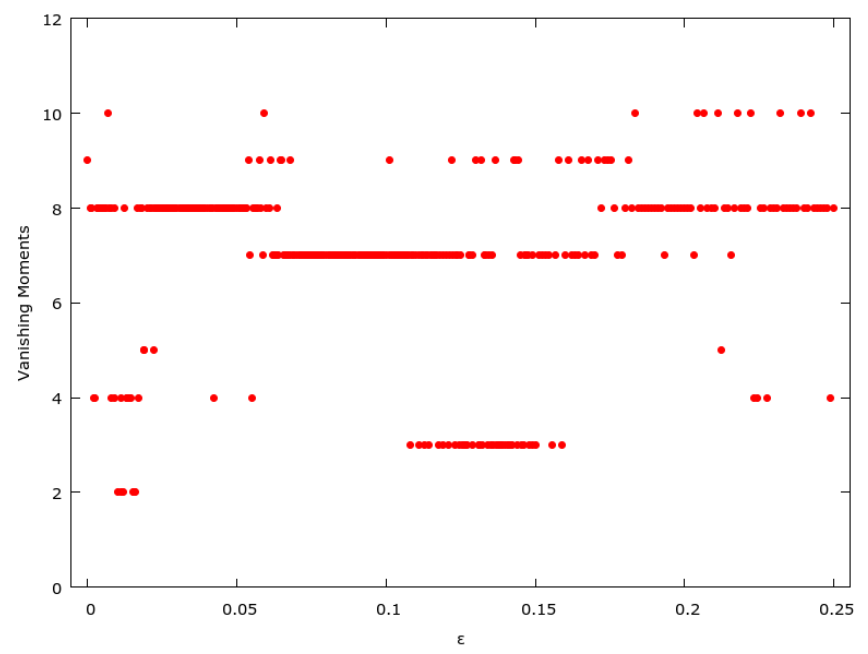

FiguRE 6. The dot at level $p$ above an $\varepsilon$-value means that the Pearson correlation coefficient applying Algorithm 6.6 with the D2 $p$ Daubechies wavelet (with $p$ vanishing moments) is the biggest one (always greater than 0.99 and biggest in comparison with D2k Daubechies wavelets with $k \neq p$ vanishing moments).

that, not surprisingly (see Proposition 5.3), are in the interval $(0,1)$ and "far" from zero: $\mathrm{R}(\tau) \in[0.6822,0.9669]$ (see the right part of Figure 5).

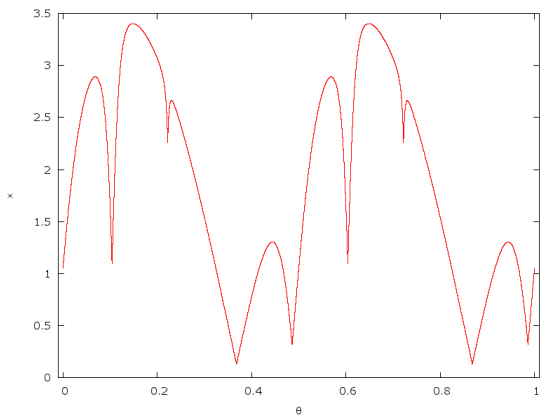

(A) Plot of the attractor of the system (15) for $\sigma=1.699219$ (and $\varepsilon=$ $0.039688)$.

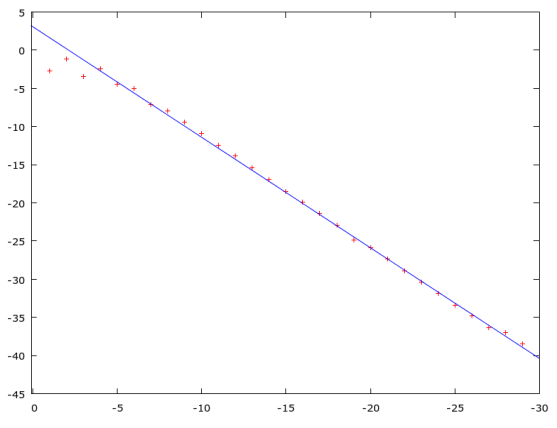

(B) Plot of the pairs $\left(j, s_{j}\right)(-29 \leq j<$ 0 ) for this attractor in red and their associated linear model in blue (with $-29 \leq j \leq-4)$.

FiguRE 7. According to Remark 4.5, the numerical value of the regularity of the attractor of system (15) based on the pairs $\left(j, s_{j}\right)$ with $-29 \leq j \leq-4$ is $\mathrm{R}(\tau)=0.91431$.

Observe that (see the right picture of Figure 7) in agreement with the computed Pearson correlation coefficient, the model given by equation (13) is approximately linear (as we expect). Also, few of the first coefficients are not well fitted for the linear model, in concordance to Remark 4.5. In this case, possibly, the self similarity is undeveloped at these scales.

The pinched case: $\sigma \in[1,1.5]$. In this case $\varepsilon=0$. Therefore, according to Theorem 5.1 and Proposition 5.3, the attractor is pinched and, hence, discontinuous 
almost everywhere. That is, the function $\varphi$ whose graph is the attractor is an upper semi continuous function (see the left picture of Figure 8). Thus, in agreement with Proposition 5.3, the estimated regularity is equal to zero for the whole range of parameters as it can be guessed in the left part of Figure 5.

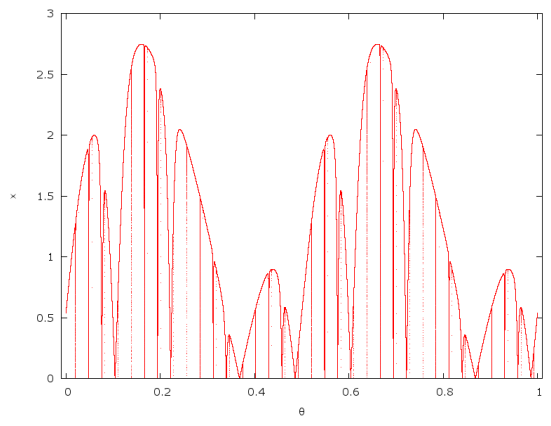

(A) Plot of the attractor of the system (15) for $\sigma=1.425781$ (and $\varepsilon=$ $0.0)$.

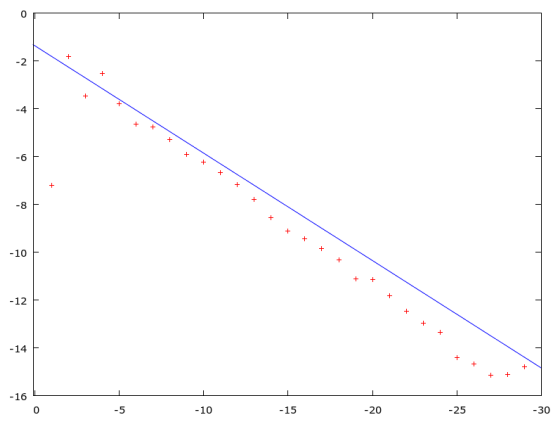

(в) Plot of the pairs $\left(j, s_{j}\right)(-29 \leq j<$ 0 ) for this attractor in red and their associated linear model in blue (with $-29 \leq j \leq-5)$.

FIGURE 8. According to Remark 4.5, the numerical value of the regularity of the attractor of system (15) based on the pairs $\left(j, s_{j}\right)$ with $-29 \leq j \leq-5$ is $\mathrm{R}(\tau)=0.0049$.

Moreover, even when the hypothesis of Lemma 2.3 are not verified the coefficients obtained are almost linear. Indeed, from Theorem 4.2 , the model given by equation (13) has more freedom because there is a gap in $[-1 / 2,1 / 2]$ (see Theorem 4.2). However, see the right picture of Figure 8, the model is linear (except for a few first values as before). This means that the proposed solutions of Problem (1) and (2) does not add error on our computations (as the case of the left picture of Figure 8).

Approaching pinching case: $\sigma \in(1.5, \widetilde{\sigma})$. In this region we have $\varepsilon=(\sigma-1.5)^{2} \lesssim$ $7.29 \times 10^{-4}$. That is, we are "close" to the pinched case. Therefore, since $g_{\varepsilon}=$ $\varepsilon+|\cos (2 \pi \theta)|$ is Lipschitz, by Proposition 5.3, the regularity must have a jump from 0 to 1 . Thus, in the estimated regularities one must perceive such jump. Having said that, the estimated regularities in this region are not so good (see the caption of Figure 9). However, looking at Figure 5 and 9, the pass from 0 to 1 in a "fast way" is still observed.

Observe that, as in the other cases, the first few values of the suprema $s_{j}$ are the worst fitted (see the right pictures in Figure 9). But, in contrast with the previous regions, the rest of values of $s_{j}$ are not so well behaved. Nevertheless, they have a big Pearson correlation coefficient. This bad behaviour is probably due to a big value of the constant $C>0$ of Theorem 4.2. Indeed, for such range of values, we are close to the change of space from positive regularity to zero. That is, from Remark 4.3 the constant $C>0$ depends on $\varepsilon$ and $\sigma$, and Figure 10 shows how $C$ depends on $\varepsilon$ and $\sigma=1.5$.

Finally, recall that the Fast Wavelet Transform is not the unique way to obtain the wavelet coefficients. In a forthcoming paper we will explore the technique of solving numerically the Invariance Equation given in Remark 6.7 which can be more adapted to the dynamical complexity of the object and to better recover the large set of zeros of the SNA. Moreover, it is also interesting to explore other models and understand other "routes to complexity" for the SNA's. In particular the study of 


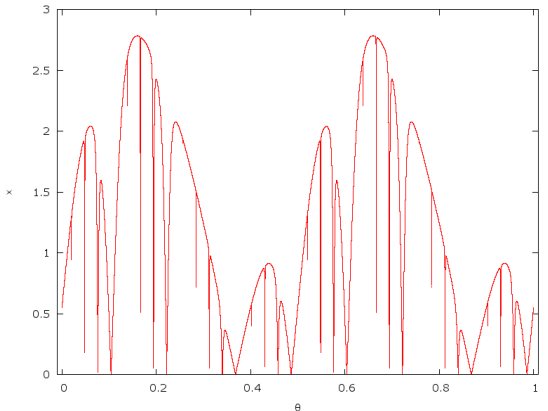

(A) Plot of the attractor of the system (15) for $\sigma=1.513672$ (and $\varepsilon=$ $0.000187)$.

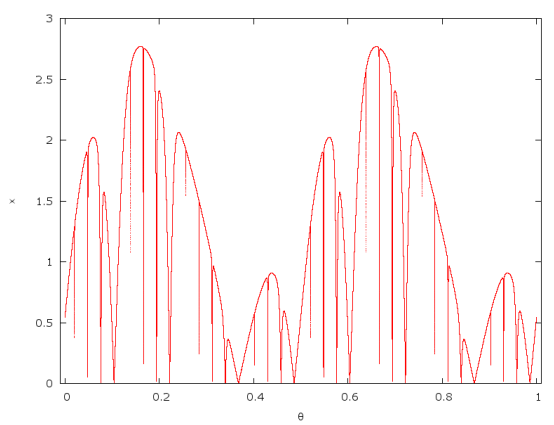

(C) Plot of the attractor of the system (15) for $\sigma=1.507812$ (and $\varepsilon=$ $0.000061)$.

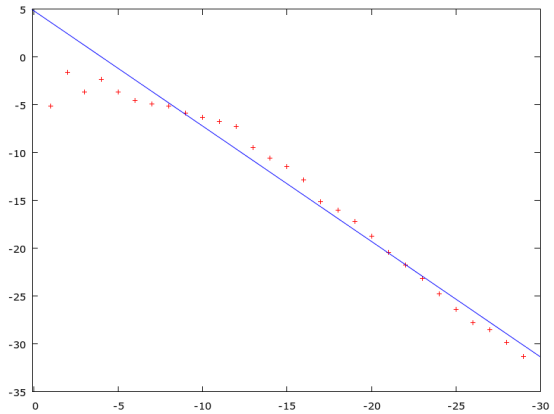

(B) Plot of the pairs $\left(j, s_{j}\right)(-29 \leq j<$ $0)$ for this attractor in red and their associated linear model in blue (with $-29 \leq j \leq-5)$.

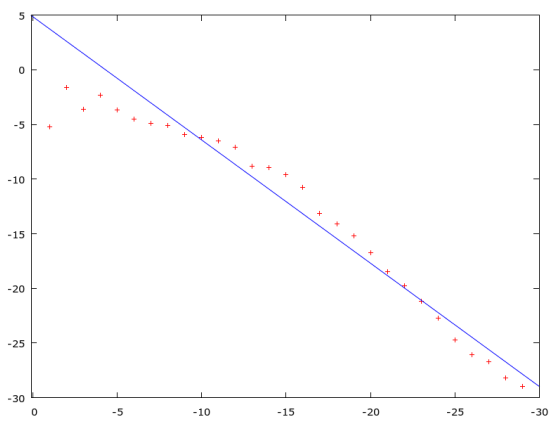

(D) Plot of the pairs $\left(j, s_{j}\right)(-29 \leq j<$ 0 ) for this attractor in red and their associated linear model in blue (with $-29 \leq j \leq-5)$.

Figure 9. According to Remark 4.5, the numerical value of the regularity of the attractor of system (15) based on the pairs $\left(j, s_{j}\right)$ with $-29 \leq j \leq-5$ is, for these two instances of $\sigma \in(1.5, \widetilde{\sigma})$, is $\mathrm{R}(\tau)=0.6266$ and $\mathrm{R}(\tau)=0.4951$ respectively

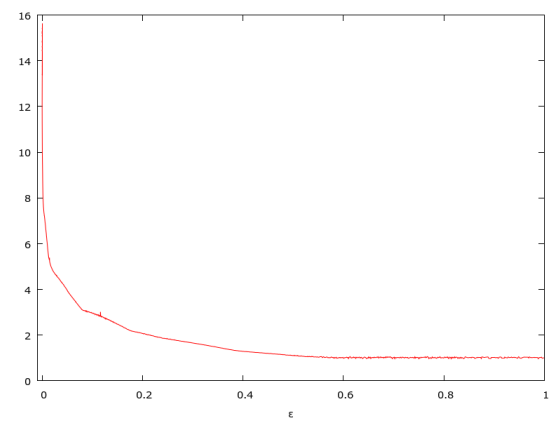

Figure 10. The plot of the "functional space jump", in terms of the constant $C$ from equation (13), for the system (15) when $\varepsilon \in(0,1]$ and $\sigma=1.5$.

the arc length curve (see [JT08]) or the Hausdorff dimension (see [GJ13]) by means of wavelets can help understanding some of these routes to strangeness. 
Appendix. On the Construction of the $\mathcal{C}^{2}$ Diffeomorphism $h$ : A Degree ONE MONOTONE SPLINE ON THE CIRCLE

This appendix is devoted to construct the diffeomorphism $h: \mathbb{S}^{1} \longrightarrow \mathbb{S}^{1}$ as a $\mathcal{C}^{2}$ degree one rational quadratic monotone spline on the circle following [Del83].

To construct the map $h$ as a $\mathcal{C}^{2}$ rational quadratic monotone spline we will start by listing the conditions that this map must satisfy. To this end we introduce the following notation. We set $x_{i}:=\frac{i}{N}$ for $i=0, \ldots, N ; \theta_{i}:=\widetilde{\theta}_{i}$ for $i=0, \ldots, N-1$ and $\theta_{N}:=1+\widetilde{\theta}_{0}=1+\theta_{0}$ (recall that $N=2^{J}$ ). Also, as usual when dealing with splines, we will set $\left.h\right|_{\left[x_{i}, x_{i+1}\right]}=h_{i}$ for $i=0,1, \ldots, N-1$.

(i) The interpolation condition:

$h\left(x_{i}\right)=h_{i}\left(x_{i}\right)=\theta_{i}$ for $i=0, \ldots, N-1 \quad$ and $\quad h\left(x_{N}\right)=h_{N-1}\left(x_{N}\right)=\theta_{N}$.

This is the basic requirement on the map $h$ from Definition 6.1. Observe also that the condition $h\left(x_{N}\right)=\theta_{N}$ already implies that $h$ has degree one because

$$
h(1)=h\left(x_{N}\right)=\theta_{N}=\theta_{0}+1=h\left(x_{0}\right)+1=h(0)+1 .
$$

(ii) The continuity condition:

$$
h_{i}\left(x_{i+1}\right)=h_{i+1}\left(x_{i+1}\right) \text { for } i=0, \ldots, N-2 .
$$

(iii) The $\mathcal{C}^{1}$ condition:

$$
\begin{aligned}
& h_{i}^{\prime}\left(x_{i+1}\right)=h_{i+1}^{\prime}\left(x_{i+1}\right):=d_{i+1} \text { for } i=0, \ldots, N-2 \text { and } \\
& \qquad d_{N}:=h_{N-1}^{\prime}\left(x_{N}\right)=h_{0}^{\prime}\left(x_{0}\right):=d_{0} .
\end{aligned}
$$

(iv) The $\mathcal{C}^{2}$ condition:

$$
h_{i}^{\prime \prime}\left(x_{i+1}\right)=h_{i+1}^{\prime \prime}\left(x_{i+1}\right) \text { for } i=0, \ldots, N-2 \text { and } \quad h_{N-1}^{\prime \prime}\left(x_{n}\right)=h_{0}^{\prime \prime}\left(x_{0}\right) .
$$

To construct the spline $h$ verifying these conditions, for $\theta \in\left[x_{i}, x_{i+1}\right]$ we define

$$
h_{i}(\theta):=\frac{\theta_{i+1} \vartheta^{2}+\Delta_{i}^{-1}\left(\theta_{i+1} d_{i}+\theta_{i} d_{i+1}\right) \vartheta(1-\vartheta)+\theta_{i}(1-\vartheta)^{2}}{\vartheta^{2}+\Delta_{i}^{-1}\left(d_{i}+d_{i+1}\right) \vartheta(1-\vartheta)+(1-\vartheta)^{2}},
$$

where $\vartheta=N\left(\theta-x_{i}\right), \Delta_{i}=N\left(\theta_{i+1}-\theta_{i}\right)$ and the derivatives $d_{i}$ are the unknowns.

As it can be seen in [Del83], the fact that $0 \leq \theta_{i}<\theta_{j}<1 \leq \theta_{N}$ whenever $0 \leq i<j<N$ implies that the derivatives $d_{i}$ are positive and, hence,

$$
h_{i}^{\prime}(\theta)=\frac{d_{i+1} \vartheta^{2}+2 \Delta_{i} \vartheta(1-\vartheta)+d_{i}(1-\vartheta)^{2}}{\left(\vartheta^{2}+\Delta_{i}^{-1}\left(d_{i}+d_{i+1}\right) \vartheta(1-\vartheta)+(1-\vartheta)^{2}\right)^{2}}
$$

is positive for every $\theta \in\left[x_{i}, x_{i+1}\right]$. Thus, the map $h$ defined by equation (23) is strictly monotone (and hence a diffeomorphism) on $\mathbb{S}^{1}$.

Now, we will show how to compute the unknowns $d_{i}$ to determine the spline $h$, how to show that the $d_{i}$ are indeed positive and, finally, how to globally bound $h^{\prime}$. To this end observe that by computing $h^{\prime \prime}$ from equation (24), condition (iv) gives

$$
d_{i}\left(-2+a_{i-1} d_{i-1}+\left(a_{i-1}+a_{i}\right) d_{i}+a_{i} d_{i+1}\right)=b_{i}
$$

for $i=1, \ldots, N-1$, where $a_{i}:=\frac{1}{\Delta_{i}}$ and $b_{i}:=\Delta_{i-1}+\Delta_{i}$. In a similar way, $h_{N-1}^{\prime \prime}\left(x_{N}\right)=h_{0}^{\prime \prime}\left(x_{0}\right)$ and $d_{N}=d_{0}$ give

$$
\frac{d_{0}^{2}}{\Delta_{N-1}}+\frac{d_{0}^{2}}{\Delta_{0}}+\frac{d_{N-1} d_{0}}{\Delta_{N-1}}+\frac{d_{1} d_{0}}{\Delta_{0}}-2 d_{0}-\Delta_{N-1}-\Delta_{0}=0 .
$$


Thus, by using again that $d_{N}=d_{0}$, the unknowns $d_{i}$ verify the following non-linear system of equations

$$
\left\{\begin{aligned}
d_{0}= & \frac{1}{2\left(a_{N-1}+a_{0}\right)}\left(2-a_{N-1} d_{N-1}-a_{0} d_{1}+\right. \\
& \left.\sqrt{\left(2-a_{N-1} d_{N-1}-a_{0} d_{1}\right)^{2}+4\left(a_{N-1}+a_{0}\right) b_{N}}\right) \\
d_{i}= & \frac{1}{2\left(a_{i-1}+a_{i}\right)}\left(2-a_{i-1} d_{i-1}-a_{i} d_{i+1}+\quad \text { for } i=1, \ldots, N-2\right. \\
d_{N-1}= & \frac{1}{2\left(a_{N-2}+a_{N-1}\right)}\left(2-a_{N-2} d_{N-2}-a_{N-1} d_{0}+\right. \\
\sqrt{\left(2-a_{N-2} d_{N-2}-a_{N-1} d_{0}\right)^{2}+4\left(a_{N-2}+a_{N-1}\right) b_{N-1}} &
\end{aligned}\right.
$$

where we have defined $b_{N}=\Delta_{N-1}+\Delta_{0}$. To solve this system is equivalent to find a fixed point $\mathbf{d}=G(\mathbf{d})$ where $G=\left(G_{0}, \ldots, G_{N-1}\right): \mathbb{R}^{N} \longrightarrow \mathbb{R}^{N}$ is the map defined by

$$
\left\{\begin{aligned}
G_{0}(\mathbf{d})= & \frac{1}{2\left(a_{N-1}+a_{0}\right)}\left(2-a_{N-1} d_{N-1}-a_{0} d_{1}+\right. \\
& \left.\sqrt{\left(2-a_{N-1} d_{N-1}-a_{0} d_{1}\right)^{2}+4\left(a_{N-1}+a_{0}\right) b_{N}}\right), \\
G_{i}(\mathbf{d})= & \frac{1}{2\left(a_{i-1}+a_{i}\right)}\left(2-a_{i-1} d_{i-1}-a_{i} d_{i+1}+\quad \text { for } i=1, \ldots, N-2,\right. \\
G_{N-1}(\mathbf{d}) & =\frac{1}{2\left(a_{N-2}+a_{N-1}\right)}\left(2-a_{N-2} d_{N-2}-a_{N-1} d_{0}+\right. \\
\sqrt{\left(2-a_{N-2} d_{N-2}-a_{N-1} d_{0}\right)^{2}+4\left(a_{N-2}+a_{N-1}\right) b_{N-1}} &
\end{aligned}\right.
$$

and $\mathbf{d}:=\left(d_{0}, d_{1}, \ldots, d_{N-1}\right) \in \mathbb{R}^{N}$.

In [Del83] it is shown that $G$ is a contracting map in the domain $\prod_{i=0}^{N-1}\left[\alpha_{i}, \beta_{i}\right]$, where

$$
\begin{gathered}
\alpha_{0}=\frac{1}{2\left(a_{N-1}+a_{0}\right)}\left(2-a_{N-1} \beta_{N-1}-a_{0} \beta_{1}+\right. \\
\left.\sqrt{\left(2-a_{N-1} \beta_{N-1}-a_{0} \beta_{1}\right)^{2}+4\left(a_{N-1}+a_{0}\right) b_{N}}\right)>0, \\
\alpha_{i}=\frac{1}{2\left(a_{i-1}+a_{i}\right)}\left(2-a_{i-1} \beta_{i-1}-a_{i} \beta_{i+1}+\quad \text { for } i=1, \ldots, N-2,\right. \\
\left.\sqrt{\left(2-a_{i-1} \beta_{i-1}-a_{i} \beta_{i+1}\right)^{2}+4\left(a_{i-1}+a_{i}\right) b_{i}}\right)>0
\end{gathered}
$$




$$
\begin{aligned}
\alpha_{N-1}= & \frac{1}{2\left(a_{N-2}+a_{N-1}\right)}\left(2-a_{N-2} \beta_{N-2}-a_{N-1} \beta_{0}+\right. \\
& \left.\sqrt{\left(2-a_{N-2} \beta_{N-2}-a_{N-1} \beta_{0}\right)^{2}+4\left(a_{N-2}+a_{N-1}\right) b_{N-1}}\right)>0, \\
\beta_{0}=\eta\left(\Delta_{N-1}, \Delta_{0}\right), & \text { for } i=1, \ldots, N-2, \\
\beta_{i}=\eta\left(\Delta_{i-1}, \Delta_{i}\right) \quad & \beta_{N-1}=\eta\left(\Delta_{N-2}, \Delta_{N-1}\right),
\end{aligned}
$$

and $\eta:(0, \infty) \times(0, \infty) \longrightarrow(0, \infty)$ is the map defined by

$$
\eta(x, y):=\frac{1}{\frac{1}{x}+\frac{1}{y}}\left(1+\sqrt{1+\left(\frac{1}{x}+\frac{1}{y}\right)(x+y)}\right) .
$$

Hence, $G$ has a unique fixed point in $\prod_{i=0}^{N-1}\left[\alpha_{i}, \beta_{i}\right]$, which is the positive solution of system (26). This shows the existence of the $\mathcal{C}^{2}$ diffeomorphism $h$ verifying the conditions from Definition 6.1 (condition (i)).

Finally we need to obtain an upper estimate of $h^{\prime}(\theta)$. To do this we will use the following auxiliary family of parabolas:

$$
D_{A}(\vartheta):=\vartheta^{2}+A \vartheta(1-\vartheta)+(1-\vartheta)^{2},
$$

where $\vartheta \in[0,1]$ and $0 \leq A<\infty$. Clearly, for every $0 \leq A<\infty, D_{A}(0)=D_{A}(1)=1$ and $D_{A}$ has a critical point at $\vartheta=1 / 2$. Moreover, this critical point is a minimum when $A \leq 2$ and a maximum when $A>2$. Also, $D_{A_{1}}(\vartheta)<D_{A_{2}}(\vartheta)$ for every $\vartheta \in(0,1)$ and $A_{1}<A_{2}$. Thus, for every $\vartheta \in[0,1]$ and $0 \leq A<\infty$,

$$
\begin{aligned}
\frac{1}{2}=D_{0}\left(\frac{1}{2}\right) \leq D_{0}(\vartheta) & \leq D_{A}(\vartheta) \\
& \leq\left\{\begin{array}{ll}
D_{2}(\vartheta)=1 & \text { if } A \leq 2 \\
D_{A}\left(\frac{1}{2}\right)=\frac{A+2}{4} & \text { if } A>2
\end{array}\right\}=\max \left\{1, \frac{A+2}{4}\right\} .
\end{aligned}
$$

The solution of system (26) is the vector

$$
\mathbf{d}:=\left(d_{0}, d_{1}, \ldots, d_{N-1}\right) \in \prod_{i=0}^{N-1}\left[\alpha_{i}, \beta_{i}\right] .
$$

Hence, for every $i=0,1, \ldots, N-1$,

$$
0<\alpha_{i} \leq d_{i} \leq \beta_{i} \leq \beta:=\max _{0 \leq i \leq N-1} \beta_{i} .
$$

So, from equation (24) we get

$$
\begin{aligned}
h_{i}^{\prime}(\theta) & =\frac{d_{i+1} \vartheta^{2}+2 \Delta_{i} \vartheta(1-\vartheta)+d_{i}(1-\vartheta)^{2}}{\left(\vartheta^{2}+\Delta_{i}^{-1}\left(d_{i}+d_{i+1}\right) \vartheta(1-\vartheta)+(1-\vartheta)^{2}\right)^{2}} \\
& \leq \frac{\beta \vartheta^{2}+2 \Delta_{i} \vartheta(1-\vartheta)+\beta(1-\vartheta)^{2}}{1 / 4}(\text { by }(27)) \\
& =4 \beta\left(\vartheta^{2}+\frac{2 \Delta_{i}}{\beta} \vartheta(1-\vartheta)+(1-\vartheta)^{2}\right) \\
& \leq 4 \beta \max \left\{1, \frac{\frac{2 \Delta_{i}}{\beta}+2}{4}\right\} \quad(\text { by }(27)) \\
& =\max \left\{4 \beta, 2\left(\Delta_{i}+\beta\right)\right\} .
\end{aligned}
$$


For each $\ell \in\{0,1, \ldots, N-1\}$ consider the interval $U_{\ell}=\left[\theta_{\ell}, \theta_{\ell}+\frac{3}{N}\right]$. By the Birkhoff Ergodic Theorem we know that there exists $\Lambda \in \mathbb{N}$ such that

$$
\left|\frac{1}{n} \sharp\left(\left\{R_{\omega}^{i}(\theta): 0 \leq i \leq n-1\right\} \cap U_{\ell}\right)-\frac{3}{N}\right|=\left|\frac{1}{n} \sum_{i=0}^{n-1} \chi_{U_{\ell}}\left(R_{\omega}^{i}(\theta)\right)-\int_{\mathbb{S}^{1}} \chi_{U_{\ell}} d \theta\right|<\frac{1}{N}
$$

for all $n \geq \Lambda$, where $\chi_{U_{\ell}}(\cdot)$ is the characteristic function of the interval $U_{\ell}$. Therefore,

$$
2 \leq \frac{N}{n} \sharp\left(\left\{R_{\omega}^{i}(\theta): 0 \leq i \leq n-1\right\} \cap U_{\ell}\right) .
$$

Assuming that $N$ is large enough ${ }^{2}$ so that we can take $n=N$, since the data $\theta_{\ell}$ is sorted, it follows that $\theta_{\ell+1} \in U_{\ell}$. Hence, $\theta_{\ell+1}-\theta_{\ell} \leq \frac{3}{N}$ and, consequently, $\Delta_{\ell}=N\left(\theta_{\ell+1}-\theta_{\ell}\right) \leq 3$. Now, we claim that

$$
\beta \leq \frac{3(1+\sqrt{5})}{2} \text {. }
$$

Hence, by the claim,

$$
h_{i}^{\prime}(\theta) \leq \max \left\{4 \beta, 2\left(\Delta_{i}+\beta\right)\right\} \leq 6(1+\sqrt{5})
$$

for every $i=0,1, \ldots, N-1$. Thus,

$$
\|h\|_{\mathcal{C}^{1}} \leq 1+\max _{0 \leq i \leq N-1}\left\|h_{i}^{\prime}\right\|_{\infty} \leq 1+6(1+\sqrt{5})<7(1+\sqrt{5})
$$

as stated in equation (19).

To end the appendix we only have to prove the claim that $\beta \leq \frac{3(1+\sqrt{5})}{2}$. To this end we will rewrite the map $\eta$ in a more appropriate way:

$$
\begin{aligned}
\eta(x, y) & =\frac{1}{\frac{1}{x}+\frac{1}{y}}\left(1+\sqrt{1+\left(\frac{1}{x}+\frac{1}{y}\right)(x+y)}\right) \\
& =\frac{1}{\frac{1}{x}+\frac{1}{y}}+\sqrt{\frac{1}{\left(\frac{1}{x}+\frac{1}{y}\right)^{2}}+\frac{x+y}{\frac{1}{x}+\frac{1}{y}}} \\
& =\frac{1}{\frac{1}{x}+\frac{1}{y}}+\sqrt{\left(\frac{1}{\left.\frac{1}{x}+\frac{1}{y}\right)^{2}+x y .}\right.}
\end{aligned}
$$

Clearly, $\frac{1}{\frac{1}{x}+\frac{1}{y}}$ and $x y$ are increasing on each variable. Consequently, $\eta(x, y)$ is increasing in both variables and, hence,

$$
\beta=\max _{0 \leq i \leq N-1} \beta_{i}=\max _{0 \leq i \leq N-1} \eta\left(\Delta_{i-1(\bmod N)}, \Delta_{i}\right) \leq \eta(3,3)=\frac{3(1+\sqrt{5})}{2}
$$

because $\Delta_{i} \leq 3$ for $i \in\{0,1, \ldots, N-1\}$.

\section{REFERENCES}

[BL76] Jöran Bergh and Jörgen Löfström. Interpolation spaces. An introduction. SpringerVerlag, Berlin, 1976. Grundlehren der Mathematischen Wissenschaften, No. 223.

[Coh03] Albert Cohen. Numerical analysis of wavelet methods, volume 32 of Studies in Mathematics and its Applications. North-Holland Publishing Co., Amsterdam, 2003.

[Del83] J. A. Delbourgo, R.; Gregory. $c^{2}$ rational quadratic spline interpolation to monotonic data. IMA Journal of Numerical Analysis, 3, 1983.

[dlLP02] Rafael de la Llave and Nikola P. Petrov. Regularity of conjugacies between critical circle maps: an experimental study. Experiment. Math., 11(2):219-241, 2002.

\footnotetext{
${ }^{2}$ There is no evidence that we can take $n=N$ large enough in the above computation but there is numerical evidence that $\theta_{\ell+1}-\theta_{\ell} \leq \frac{2}{N}$ for every $\ell=0,1, \ldots, N-1$.
} 
[Fra99] Michael W. Frazier. An introduction to wavelets through linear algebra. Undergraduate Texts in Mathematics. Springer-Verlag, New York, 1999.

[GJ13] M. Gröger and T. Jäger. Dimensions of Attractors in Pinched Skew Products. Comm. Math. Phys., 320(1):101-119, 2013.

[GOPY84] Celso Grebogi, Edward Ott, Steven Pelikan, and James A. Yorke. Strange attractors that are not chaotic. Phys. D, 13(1-2):261-268, 1984.

[HW96] Eugenio Hernández and Guido Weiss. A first course on wavelets. Studies in Advanced Mathematics. CRC Press, Boca Raton, FL, 1996. With a foreword by Yves Meyer.

[Jor01] Àngel Jorba. Numerical computation of the normal behaviour of invariant curves of n-dimensional maps. Nonlinearity, 14(5):943-976, 2001.

[JT08] Àngel Jorba and Joan Carles Tatjer. A mechanism for the fractalization of invariant curves in quasi-periodically forced 1-D maps. Discrete Contin. Dyn. Syst. Ser. B, 10(2-3):537-567, 2008.

[Kel96] Gerhard Keller. A note on strange nonchaotic attractors. Fund. Math., 151(2):139-148, 1996.

[Mal98] Stéphane Mallat. A wavelet tour of signal processing. Academic Press Inc., San Diego, CA, 1998.

[Mey01] Yves Meyer. Oscillating patterns in image processing and nonlinear evolution equations, volume 22 of University Lecture Series. American Mathematical Society, Providence, RI, 2001. The fifteenth Dean Jacqueline B. Lewis memorial lectures.

[RS96] Thomas Runst and Winfried Sickel. Sobolev spaces of fractional order, Nemytskij operators, and nonlinear partial differential equations, volume 3 of de Gruyter Series in Nonlinear Analysis and Applications. Walter de Gruyter \& Co., Berlin, 1996.

[Sta97] Jaroslav Stark. Invariant graphs for forced systems. Phys. D, 109(1-2):163-179, 1997. Physics and dynamics between chaos, order, and noise (Berlin, 1996).

[Sta99] Jaroslav Stark. Regularity of invariant graphs for forced systems. Ergodic Theory Dynam. Systems, 19(1):155-199, 1999.

[Ste70] Elias M. Stein. Singular integrals and differentiability properties of functions. Princeton Mathematical Series, No. 30. Princeton University Press, Princeton, N.J., 1970.

[Tri83] Hans Triebel. Theory of function spaces, volume 78 of Monographs in Mathematics. Birkhäuser Verlag, Basel, 1983.

[Tri92] Hans Triebel. Theory of function spaces. II, volume 84 of Monographs in Mathematics. Birkhäuser Verlag, Basel, 1992.

[Tri06] Hans Triebel. Theory of function spaces. III, volume 100 of Monographs in Mathematics. Birkhäuser Verlag, Basel, 2006.

Departament de Matemàtiques, Edifici CC, Universitat Autònoma de Barcelona, 08913 Cerdanyola del Vallès, Barcelona, Spain

E-mail address: alseda@mat.uab.cat, jmm@mat.uab.cat, dromero@mat.uab.cat 